\title{
DNA-Templated Aggregates of Strongly- Coupled Cyanine Dyes: Nonradiative Decay Governs Exciton Lifetimes
}

Jonathan S. Huff, ${ }^{\dagger}$ Paul H. Davis,,$^{\dagger}$ Allison Christy, ${ }^{\dagger}$ Donald L. Kellis, ${ }^{\dagger}$ Nirmala Kandadai, ${ }^{\dagger} \mathrm{Zi}$ S.D. Toa, ${ }^{\S}$ Gregory D. Scholes, ${ }^{\S}$ Bernard Yurke, ${ }^{\dagger, t}$ William B. Knowlton, ${ }^{\dagger, t}$ and Ryan D. Pensack

${ }^{\dagger}$ Micron School of Materials Science \& Engineering and Department of Electrical \& Computer Engineering, Boise State University, Boise, Idaho 83725, United States

${ }^{\S}$ Department of Chemistry, Princeton University, Princeton, New Jersey 08544, United States

\section{Table of Contents:}

Supporting Information S.1: Dye Structure, DNA Sequences, Construct Preparation, and Purification Procedure

Supporting Information S.2: Optical Spectroscopy Experimental Methods

Supporting Information S.3： Origin of Absorption Spectrum Variation: Evidence for Structural Heterogeneity in J-dimer Solution

Supporting Information S.4: Monomer Photophysics

Supporting Information S.5: Fluorescence Excitation Spectroscopy Establishes the Multicomponent Nature of Aggregate Solutions

Supporting Information S.6: Extraction of the "Pure" J-dimer Emission Spectrum

Supporting Information S.7: H-tetramer Solution Emission

Supporting Information S.8: Mathematical and Physical Justification of Biexponential Fits for Aggregate Solution Time-Resolved Fluorescence

Supporting Information S.9: Derivation of Expected Fluorescence Decay Rate for J-Aggregates

Supporting Information S.10: Transient Absorption Pulse Characterization

Supporting Information S.11: Comparison of Sample Absorption Spectra and Laser Spectra

Supporting Information S.12: Ground State Recovery of Aggregates is Independent of Excitation Wavelength

Supporting Information S.13: Ground State Recovery of Aggregates is Independent of Incident Pump Fluence

Supporting Information S.14: Quantitative Analysis of Radiative and Nonradiative Rates Supporting Information S.15: Charge Transfer is Ruled Out as a Nonradiative Decay Mechanism 


\section{Supporting Information S.1:}

1 Dye Structure, DNA Sequences, Construct Preparation, and Purification Procedure

\subsection{Dye Structure}

The dye employed in all constructs studied in this work was a custom Cy5 derivative from Integrated DNA Technologies (IDT; Coralville, IA, USA) that is functionalized with flexible propyl chain linkers attached to the nitrogens of both heterocycle rings. This functionalization scheme enables integration of the Cy5 into the DNA backbone internally via covalent bonding at both the 5' and 3' ends. The structure of the dye with attachment sites indicated is shown below in Figure S 1.1.

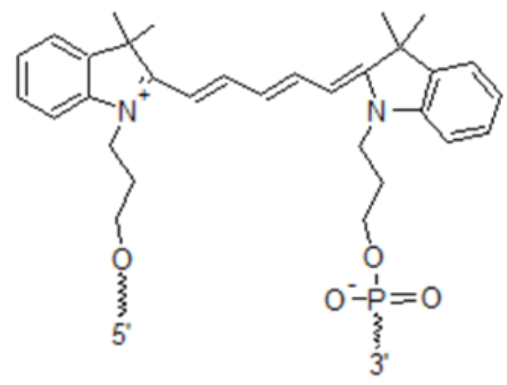

Figure S 1.1. Structure of the internal Cy5 dye including dual linkers to the DNA backbone (adapted from IDT website).

\subsection{DNA Sequences}

All constructs discussed in the main text are derived from hybridization of two complementary 26 nucleotide (nt) DNA oligomer strand sequences. These strands, herein referred to as "Cy5-A" and its complement, "Cy5-B", have Cy5 molecules internally attached within the DNA's phosphate backbone via dual covalent linkers utilizing a phosphoramidite approach as described above in Section 1.1. Furthermore, the individual complementary oligomer sequences were designed such that, when hybridized, the Cy5 molecules would inhabit the same rung of the DNA duplex. To illustrate how they hybridize, the strand sequences are presented below in Table S 1.1.

Table S 1.1. DNA Oligomer Strand Sequences

\begin{tabular}{|l|c|l|c|}
\hline Strand Name & End & Se quence & End \\
\hline Cy5-A & $5^{\prime}$ & CAGTCATAATATGCGA/iCy5/GCGATTATAT & 3 \\
\hline Cy5-B & $3^{\prime}$ & GTCAGTATTATACGCT/iCy5/CGCTAATATA & 5 \\
\hline
\end{tabular}

Note that throughout the manuscript (and for the remainder of the SI), unless otherwise noted all references to the "monomer" refer to the Cy5-A strand given that the photophysics of the Cy5-A and Cy5B monomer strands are virtually identical (see Supporting Information S.4: Monomer Photophysics). 
A detailed description of the construct preparation procedure has been reported previously, ${ }^{1}$ but a brief summary follows. DNA oligomers internally functionalized with Cy5 were purchased from IDT as a lyophilized, high-performance liquid chromatography (HPLC) purified powder. Oligomers were rehydrated with ultrapure water (Barnstead Nanopure, Thermo Scientific) to create $100 \mu \mathrm{M}$ stock solutions of Cy5-A and Cy5-B (Table S 1.1). TAE buffer (10× stock solution, $\mathrm{pH} 8.0)$ and $\mathrm{MgCl}_{2}$ (99\% purity) were purchased from Fisher Scientific. The 10× TAE stock solution was diluted to $1 \times$ (corresponding to $40 \mathrm{mM}$ tris(hydroxymethyl)aminomethane, $20 \mathrm{mM}$ acetic acid, and $1 \mathrm{mM}$ ethylenediaminetetraacetic acid) with ultrapure water, while the $\mathrm{MgCl}_{2}$ was dissolved in ultrapure water to create a $1.375 \mathrm{M}$ stock solution.

The stock solution of Cy5-A oligomer strands was used to prepare all "monomer" solutions unless otherwise explicitly stated. Hybridization of the oligomers to create DNA duplexes for the J-dimer and Htetramer constructs was accomplished by combining equimolar amounts of complementary Cy5-A and Cy5-B oligomers in a $1 \times$ TAE buffer solution to yield a final DNA concentration of 0.1-40 $\mu \mathrm{M}$. For the $\mathrm{H}$ tetramers, aliquots of the stock $\mathrm{MgCl}_{2}$ solution were added to the diluted (1×) TAE buffer solution to produce a solution with a final magnesium $\left(\mathrm{Mg}^{2+}\right)$ concentration of $100 \mathrm{mM} \mathrm{MgCl}$. Resulting solutions were then allowed to hybridize for 24 hours at room temperature.

\subsection{Purification Procedure}

To remove any excess monomer or unwanted secondary structures that formed, aggregate ( $\mathrm{J}$-dimer and $\mathrm{H}$-tetramer) constructs were purified using native polyacrylamide gel electrophoresis (PAGE). 10\% PAGE gels were cast using $1 \times$ TAE buffer with no $\mathrm{MgCl}_{2}$ added for $\mathrm{J}$-dimers and $100 \mathrm{mM} \mathrm{MgCl} 2$ added for H-tetramers. Samples were prepared at a concentration of $42 \mu \mathrm{M}$ and allowed to hybridize at room temperature for at least 24 hours. A solution with a 5:1 ratio of $6 \times$ loading buffer (New England Biolabs) to DNA sample was prepared, and the DNA/loading buffer mixtures were injected into the gels, which were allowed to run 60-90 minutes at 100-150 V. Gels were run in a $1 \times$ TAE buffer with $0 \mathrm{mM}$ and $100 \mathrm{mM}$ $\mathrm{MgCl}_{2}$ added for the J-dimer and H-tetramer solutions, respectively. After the bands were sufficiently separated from the loading buffer, the gels were imaged using a FluoroChemQ imaging system as shown in Figure S 1.2 and Figure S 1.3.
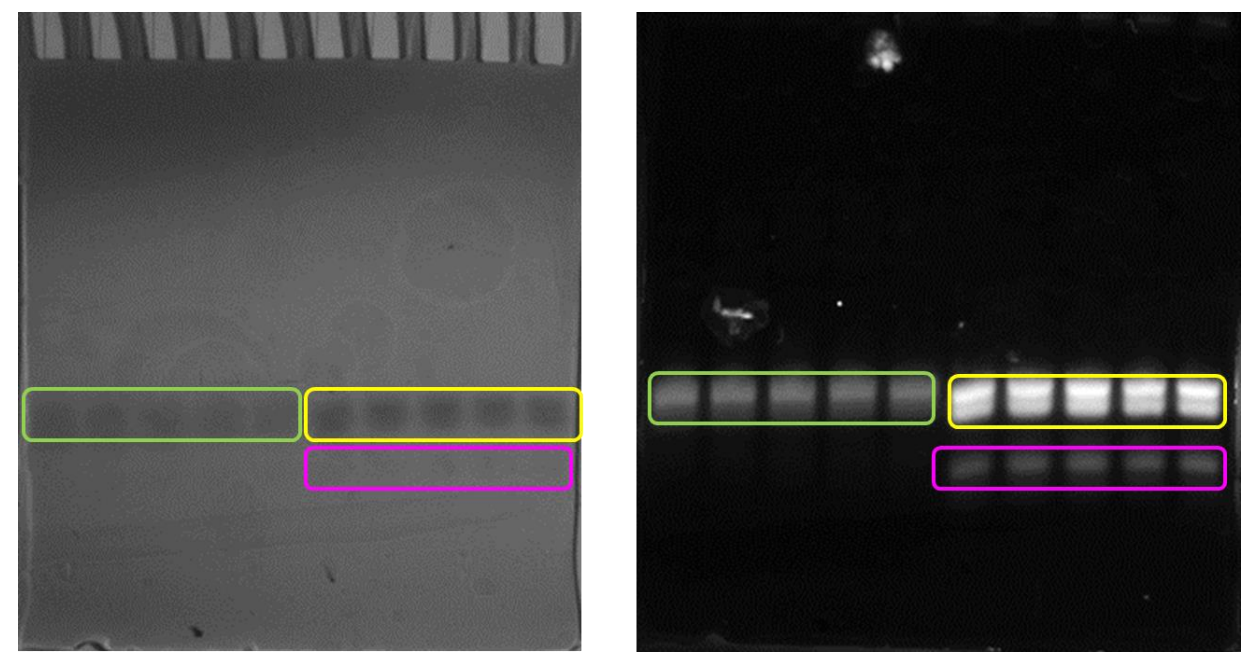

Figure S 1.2. $10 \%$ PAGE gels of J-dimer constructs run in $1 \times$ TAE buffer. The image on the left is obtained when the gel is excited with $245 \mathrm{~nm}$ light and the bands cast shadows onto an underlying phosphor plate. The image on the right is obtained when the gel 
is excited at $632 \mathrm{~nm}$ to induce fluorescence from any Cy5 containing bands. Previously PAGE-purified samples (green boxes) are compared to as-prepared (i.e., non-purified) samples (yellow boxes), which also show the presence of unhybridized single-stranded monomer (pink boxes) that is removed upon PAGE purification.

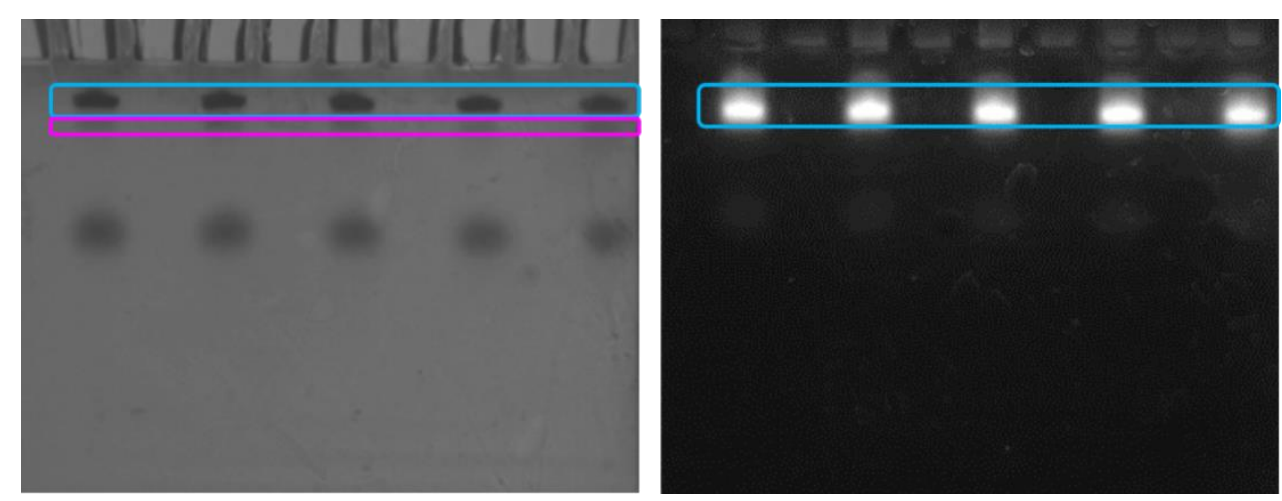

Figure S 1.3. $10 \%$ PAGE gels of H-tetramers run in $1 \times$ TAE buffer with $100 \mathrm{mMadded} \mathrm{MgCl}_{2}$. The image on the left is obtained when the gel is excited with $245 \mathrm{~nm}$ light and the bands cast shadows onto an underlying phosphor plate. The image on the right is obtained when the gel is excited at $632 \mathrm{~nm}$ to induce fluorescence from any Cy5 containing bands. The residual monomer present in the unpurified H-tetramer samples was significantly less than in the J-dimer samples (Figure S 1.2) because of the high salt concentration. Residual monomer bands (pink box) can only faintly be seen in the phosphor plate image on the left. The H-tetramer bands are indicated by the blue box. The lower unmarked band is the result of the loading buffer.

The aggregate construct bands shown in Figure S 1.2 and Figure S 1.3 were excised and redissolved in $1 \times \mathrm{TAE}$ (with $100 \mathrm{mM}$ added $\mathrm{MgCl}_{2}$ for the $\mathrm{H}$-tetramer band). The constructs were then allowed to diffuse out of the gel overnight. Once the constructs had fully diffused out of the gel and into solution, the samples were centrifuged for 1 minute at $3000 \mathrm{RCF}$ (relative centrifugal force) and extracted, with a final concentration ranging from 10-20 $\mu \mathrm{M}$, as confirmed using a NanoDrop One UV-Vis spectrophotometer (ThermoFisher Scientific; Waltham, MA, USA). The resulting PAGE-purified Htetramer sample was sufficiently concentrated when initially $20 \mu \mathrm{L}$ of sample were loaded into five separate wells, for a total of $100 \mu \mathrm{L}$ of $42 \mu \mathrm{M}$ sample. The J-dimer sample required instead the loading of $20 \mu \mathrm{L}$ of $42 \mu \mathrm{M}$ solution into 10 wells to have a resulting purified sample with high enough concentration for proper analysis. This was due to the higher concentration of monomer present at the $0 \mathrm{mM}$ added $\mathrm{MgCl}_{2}$ concentration in the J-dimer solution, which was confirmed by re-running the purified J-dimer samples and comparing them to non-purified J-dimer samples (Figure S 1.2, right panel). 


\section{Supporting Information S.2:}

\section{Optical Spectroscopy Experimental Methods}

\subsection{Sample Preparation}

All monomer and J-dimer solutions were prepared in $1 \times$ TAEbuffer $(\mathrm{pH}=8)$ with no added $\mathrm{MgCl}_{2}$, while formation of the $\mathrm{H}$-tetramer required the addition of $100 \mathrm{mM} \mathrm{MgCl}_{2}$ to the $1 \times \mathrm{TAE}$ buffer. ${ }^{1}$ As detailed below, concentrations of individual solutions were varied to achieve the appropriate optical density (OD) for each characterization technique and its associated sample cell.

\subsection{Steady-StateSpectroscopy}

All optical characterization was conducted at room temperature unless stated otherwise.

\subsubsection{UV-Vis Absorption}

A dual-beam Cary 5000 UV-Vis-NIR spectrophotometer (Agilent Technologies; Santa Clara, CA, USA) was used to acquire absorption spectra of all dye-DNA constructs. Scans were typically performed within the spectralrange of 200-800 nm, with $1 \mathrm{~nm}$ step intervals and a 0.1 second integration time. Sample solutions were diluted to a maximum optical density of 0.1 at the absorption maximum of the construct. To obtain UV-Vis absorption spectra of the monomer and aggregates, $700 \mu \mathrm{L}$ of the corresponding sample solution was pipetted into a $2 \mathrm{~mm}$ path length quartz spectrophotometer cell (Starna Cells, Inc.; Atascadero, CA, USA).

\subsubsection{Fluorescence Emission}

The monomer fluorescence emission spectrum was collected by exciting the solution in the vicinity of the Cy5 vibronic peak (ca. $600 \mathrm{~nm}$ ) using a Fluorolog-3 spectrofluorometer (HORIBA Scientific; Edison, NJ, USA). Solutions were diluted to an optical density of 0.1 or below at the absorption maximum. For the J-dimer emission spectrum extraction as well as the emission measurements of the H-tetramer presented in Supporting Information S.7:, a Horiba PTI QuantaMaster 400 spectrofluorometer (HORIBA Scientific) was used. The J-dimer solutions were excited at $595 \mathrm{~nm}$ while the H-tetramer solutions were excited at $563 \mathrm{~nm}$. For all samples, spectra were obtained from $250 \mu \mathrm{L}$ of dye-DNA sample solution pipetted into a $4 \mathrm{~mm}$ path length micro square, open-top special optical glass (SOG) fluorometer cell (Starna).

\subsubsection{Fluorescence Excitation}

Fluorescence excitation spectra of the single-stranded Cy5-A monomer and both aggregate samples were collected with a Varian Cary Eclipse fluorescence spectrophotometer. Spectra were obtained from $250 \mu \mathrm{L}$ of dye-DNA sample solution pipetted into a $4 \mathrm{~mm}$ path length micro square, open-top SOG fluorometer cell (Starna).

\subsubsection{Relative Fluorescence Quantum Yields}

\subsubsection{Monomer Fluorescence Quantum Yield}

Relative fluorescence quantum yield (FQY) measurements for the monomer were performed on a Horiba PTI QuantaMaster 400 spectrofluorometer (HORIBA Scientific). Typical scan parameters were 1 $\mathrm{nm}$ steps and $0.5 \mathrm{~s}$ integration time. The sample solution was contained in a $1 \mathrm{~cm}$ path length quartz cell (Starna) and the optical density was kept at or below 0.02 at the excitation wavelength. Oxazine- 1 in ethanol $(F Q Y=0.14)^{2}$ was used as a relative FQY standard. Absolute ethanol was used as the solvent for oxazine- 
1; a $1 \times \operatorname{TAE}\left(0 \mathrm{mM}\right.$ added $\left.\mathrm{MgCl}_{2}\right)$ buffer solution was used as the solvent for the monomer. The excitation wavelength was set to $595 \mathrm{~nm}$ and remained unchanged between measurements on the oxazine-1 and monomer solutions to ensure an equivalent intensity of light was incident on the sample for each measurement. The slit widths for the measurements were chosen to maximize the signal from the monomer while staying within the linear range of the detection system. Both the excitation and emission monochromator slit widths were set to a $2.80 \mathrm{~nm}$ bandpass. All fluorescence spectra were intensity corrected.

\subsubsection{Aggregate Solution Fluorescence Quantum Yields}

Relative FQY measurements for the aggregate solutions were performed on a Horiba Fluorolog-3 (HORIBA Scientific). Typical scan parameters were $1 \mathrm{~nm}$ steps and $0.5 \mathrm{~s}$ integration time. The sample solution was contained in a $1 \mathrm{~cm}$ path length quartz cell (Starna) and the optical density was kept at or below 0.12 at the excitation wavelength. The monomer in a $1 \times \mathrm{TAE}\left(0 \mathrm{mM}\right.$ added $\left.\mathrm{MgCl}_{2}\right)$ buffer solution $(\mathrm{FQY}=0.29$; see Supporting Information S.4: Monomer Photophysics) was used as a relative FQY standard. The same $1 \times \mathrm{TAE}\left(0 \mathrm{mM}\right.$ added $\left.\mathrm{MgCl}_{2}\right)$ buffer solution was used as the solvent for the J-dimer solution, while a $1 \times \mathrm{TAE}$ with $100 \mathrm{mM}$ added $\mathrm{MgCl}_{2}$ buffer solution was used as solvent for the $\mathrm{H}$-tetramer solution. The excitation wavelength was set to 596 and $563 \mathrm{~nm}$ for the J-dimer and H-tetramer solutions, respectively. The slit widths for the measurements were chosen to maximize the signal from the monomer reference solution while staying within the linear range of the detection system. Accordingly, for measurements on the J-dimer, both the excitation and emission monochromator slit widths were set to a $2.00 \mathrm{~nm}$ bandpass; for measurements on the H-tetramer solutions, $2.70 \mathrm{~nm}$ slit widths were employed. All fluorescence spectra were intensity corrected.

\subsection{Time-Resolved Spectroscopy \\ 2.3.1 Time Correlated Single Photon Counting}

Time-resolved fluorescence measurements were made using a DeltaFlex time correlated single photon counting (TCSPC) spectrometer (HORIBA Scientific) and a $1 \mathrm{~cm}$ path length quartz fluorometer cell (Starna). Based on the constructs' respective absorption maxima and the TCSPC spectrometer's available excitation lasers, excitation wavelengths of $653 \mathrm{~nm}$ for the monomer and J-dimer samples and $507 \mathrm{~nm}$ for the H-tetramer were chosen, with the optical density of each sample maintained at $\sim 0.1$ or less at the given excitation wavelength. Detection wavelengths for the monomer, J-dimer, and H-tetramer were $667 \mathrm{~nm}, 680 \mathrm{~nm}$, and $800 \mathrm{~nm}$, respectively. The J-dimer and H-tetramer detection wavelengths were chosen to maximize the aggregate contribution while minimizing the contribution from the subpopulation of monomers present in these samples (described in detail elsewhere) to the overall measured fluorescence emission and decay kinetics. Sample solutions were also stirred to minimize any contribution from a subpopulation of monomers. The instrument response function (IRF) was obtained by placing an aqueous Ludox colloidal silica suspension, which efficiently scatters the excitation light, into the spectrometer while keeping all settings equivalent to those used for the sample. The IRF exhibited a full-width at half maximum (FWHM) of ca. $250 \mathrm{ps,} \mathrm{which} \mathrm{is} \mathrm{representative} \mathrm{of} \mathrm{the} \mathrm{time} \mathrm{resolution} \mathrm{of} \mathrm{the} \mathrm{TCSPC} \mathrm{spectrometer} \mathrm{(see}$ Supporting Information S.10: Transient Absorption Pulse Characterization).

\subsubsection{TransientAbsorption}

Femtosecond transient absorption measurements on the constructs were performed with a $1 \mathrm{kHz}$ regeneratively amplified Ti:sapphire laser system (Coherent Mantis oscillator and Legend Elite HE USP 
amplifier; Santa Clara, CA, USA) that delivers $\sim 35 \mathrm{fs}$ pulses at $\sim 800 \mathrm{~nm}$ with an average power of $\sim 3 \mathrm{~W}$ after compression. A 50:50 beamsplitter directs approximately half of the regenerative amplifier's compressed output power to pump a femtosecond optical parametric amplifier (Coherent OPerA Solo; Light Conversion, Lithuania) that converts the $800 \mathrm{~nm}$ output of the Ti:sapphire amplifier to visible wavelengths suitable for resonant sample excitation. Pump wavelengths of 650,675 , and $565 \mathrm{~nm}$ were used for the monomer, J-dimer, and H-tetramer samples, respectively, except as noted in the case of wavelengthdependence studies (see Supporting Information S.12: Ground State Recovery of Aggregates is Independent of Excitation Wavelength).

As shown in Figure S 2.1, a 90:10 R:T beamsplitter was used to split the output of the OPA into pump and probe beams. The relative delay of the pump and probe beams was controlled by sending the pump beam down a variable path length arm controlled by a PLX OW-20-1 hollow corner cube retroreflector (PLX, Inc.; Deer Park, NY, USA) mounted on an Aerotech ALS20030 delay stage (Aerotech; Pittsburgh, PA, USA) capable of $300 \mathrm{~mm}$ travel (i.e., $2 \mathrm{~ns}$ total variable delay) and $\leq 1 \mu \mathrm{m}$ (6.7 fs) steps. The relative polarizations and powers of the pump and probe were controlled with a combination of a $\lambda / 2$ waveplate and polarizer situated in the probe beam path. All measurements were performed with pump and probe polarizations oriented at the magic angle of $54.7^{\circ}$ relative to each other at the sample, with the pump and probe beams overlapped at their foci by an off-axis parabolic reflector. The sample solutions were contained in a $2 \mathrm{~mm}$ path length quartz spectrophotometer cell (Starna), with the solutions stirred throughout all measurements. The optical density of the samples varied from ca. 0.1 to 0.4 at the excitation wavelength. The pump beam diameter was estimated to be ca. $200 \mu \mathrm{m}$ by measuring the full power of the pump beam as well as when passing through a $50 \mu \mathrm{m}$ diameter pinhole (Newport; Irvine, CA, USA). By the same technique, the probe spot size was estimated to be ca. $80 \mu \mathrm{m}$. The power of the pump and probe beams was measured with a high-sensitivity optical power sensor and meter (Coherent OP2-Vis and LabMax TOP). Incident beam fluences were controlled by a combination of neutral density filters and a $\lambda / 2$ waveplate/polarizer combination, and kept below $1 \mathrm{~mJ} / \mathrm{cm}^{2}$, unless otherwise specified (as in the case of the fluence-dependence measurements, Supporting Information S.13: Ground State Recovery of Aggregates is Independent of Incident Pump Fluence). The transient absorption signal (i.e., probe intensity after the sample) was monitored with a silicon photodiode (ThorLabs DET36A; Newton, NJ). A second DET36A Si photodiode monitored the pump beam leakage through a turning mirror in the home-built transient absorption spectrometer system to enable shot-to-shot normalization. The outputs of the two Si photodiodes were fed into a pair of SR520 gated integrators (SRS, Stanford Research Systems; Sunnyvale, CA, USA) triggered by the $1 \mathrm{kHz}$ TTL output of the Q-switch in the regenerative amplifier's Nd:YLF pump laser (Coherent Evolution). The single-shot output of the gated integrators was then fed into an SR235 analog processor (SRS), and the analog output (specifically $\ln (10 A / B)$, where $A$ is the probe beam transient absorption signal and $B$ is the pump leakage shot-to-shot normalization reference signal) was passed to an SR810 lock-in amplifier (SRS). The SR810 was phase-locked to the reference output from a MC2000 optical chopper (ThorLabs) equipped with an MC1F10 blade that chopped the pump beam at a frequency of $500 \mathrm{~Hz}$ to enable differential measurements of the $1 \mathrm{kHz}$ probe beam intensity in the presence and absence of the pump beam on consecutive shots. The chopper, in turn, was triggered by the $1 \mathrm{kHz}$ TTL output of the Nd:YLF pump laser Q-switch and down-converted to $f / 2$ (i.e., $500 \mathrm{~Hz}$ ). The lock-in time constant was typically set to 30 or $100 \mathrm{~ms}$, with a minimum $5 \times$ dwell time at each data point, plus $60 \mathrm{~Hz}$ and $120 \mathrm{~Hz}$ filters enabled to minimize electrical noise. A/D conversion of the lock-in output was accomplished by a 16-bit NI-9215 data acquisition board mounted in a cDAQ-9171 chassis (National 
Instruments; Austin, TX, USA). Depending on signal level, multiple scans were acquired and averaged to improve signal-to-noise ratios.

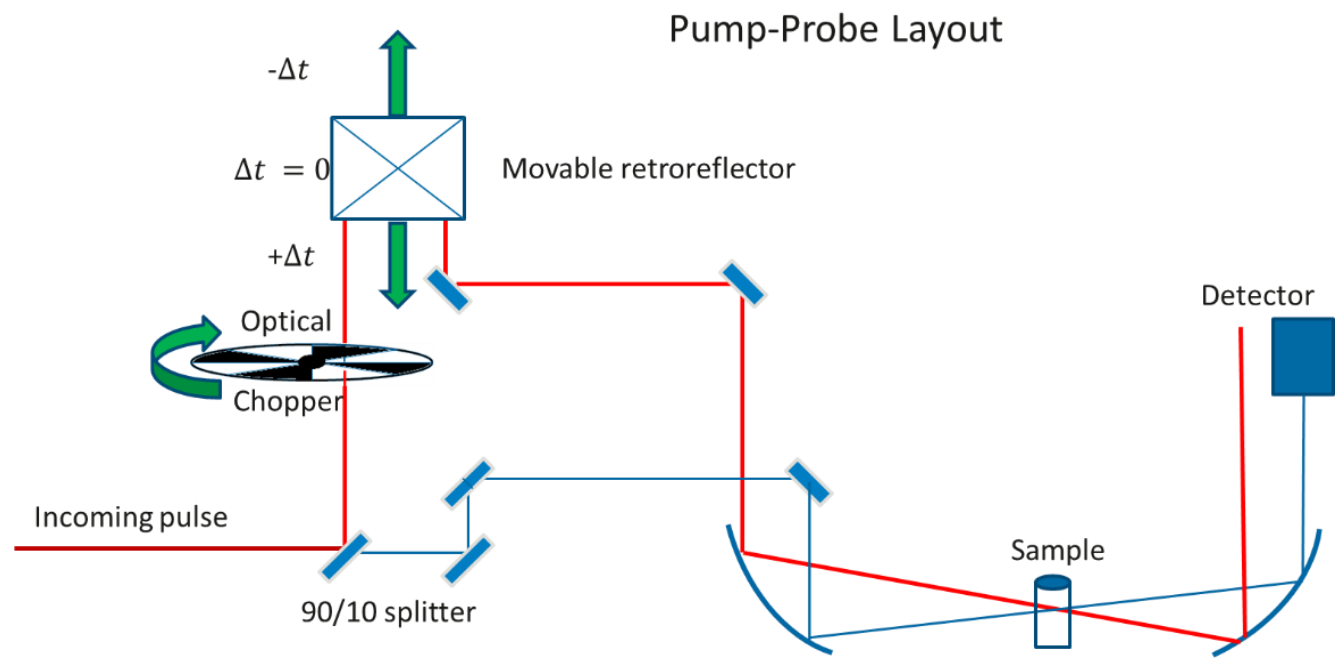

Figure S 2.1. Schematic diagram of the transient absorption (TA) spectrometer optical configuration. 


\section{Supporting Information S.3:}

\section{Origin of Absorption Spectrum Variation: Evidence for Structural}

Heterogeneity in J-dimer Solution

In the current supporting section, we discuss the origin of the variation of the absorption spectrum of the J-dimer solution and present evidence for structural heterogeneity being responsible for these variations.

Figure S.3.1 displays the absorption spectrum of a sample prior to PAGE purification (blue trace, labeled "unpurified" from here on), a PAGE purified sample reported by Cannon et al., ${ }^{1}$ and a PAGEpurified sample reported in this work. As can be seen from Fig S.3.1, the unpurified sample exhibits good agreement with the PAGE-purified sample reported by Cannon et al. The PAGE-purified sample reported in this work, in contrast, is similar, but exhibits distinguishable differences. Notably, the peak position and linewidth of the primary absorption feature has not changed, indicating that the major J-dimer population responsible for this absorption feature has not changed. The most significant spectral difference in the current work is that the ratio of the vibronic absorption bands peaking at 666 and $594 \mathrm{~nm}$ have changed considerably in the PAGE-purified sample. Specifically, the amplitude of the band peaking at $594 \mathrm{~nm}$ in the PAGE-purified sample reported in this work is considerably larger than that of the unpurified sample and the PAGE-purified sample reported by Cannon et al.

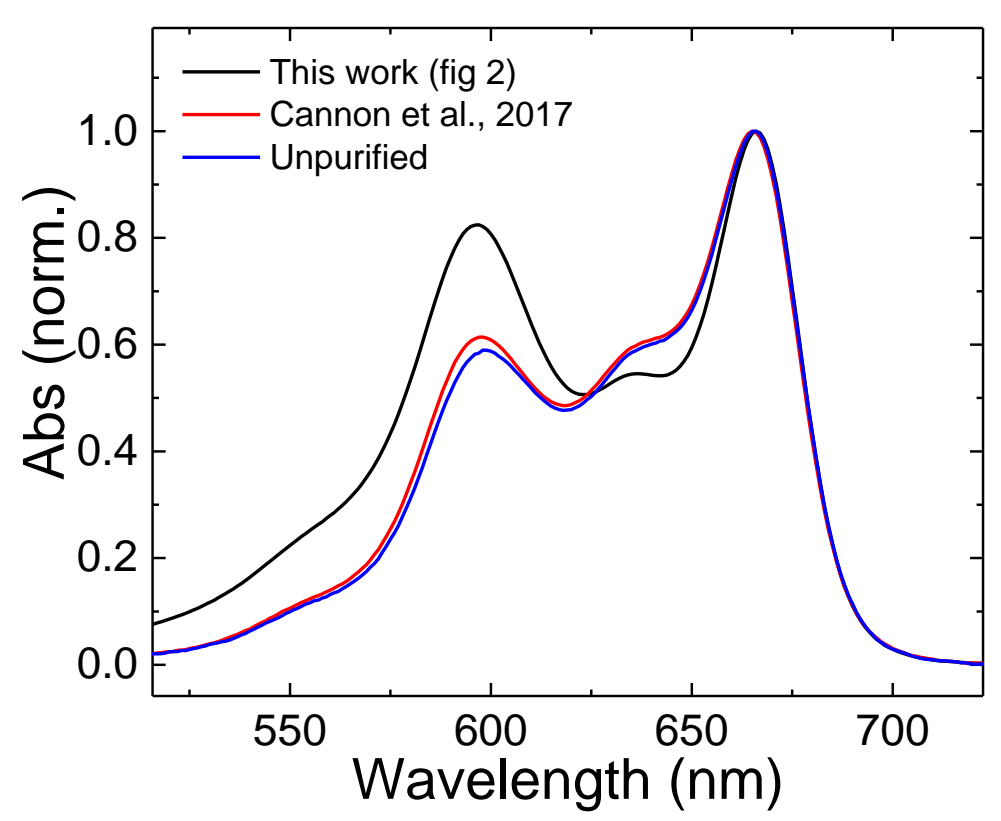

Fig S.3.1. Absorption spectra of the unpurified J-dimer solution in the present work (blue), PAGE-purified solution of Cannon et al. ${ }^{1}$ (red), and PAGE-purified solution in the present work (black). 
To investigate the origin of the different J-dimer solution absorption spectra, temperaturedependent absorption measurements were performed. Figure S.3.2 displays the absorption spectra collected for an unpurified J-dimer solution. The solution was first heated to $70^{\circ} \mathrm{C}$ to melt the duplex DNA. Consistent with our expectations, panel A shows that the absorption spectrum at $70^{\circ} \mathrm{C}$, where primarily single strand DNA is present, strongly resembles that of the monomer. As the solution is cooled through the DNA denaturation temperature, the absorbance spectrum begins to deviate from that of the monomer. Specifically, panel B shows that at $54^{\circ} \mathrm{C}$, just below the single strand (ss)-double strand (ds) transition, an absorption feature appears at $594 \mathrm{~nm}$, while features that would be consistent with the J-dimer, such as the strong absorption at $666 \mathrm{~nm}$, are notably absent. Additionally, panel C shows that, in the 604 to $614 \mathrm{~nm}$ spectral range, two isosbestic points are observed (at 607.5 and $610 \mathrm{~nm}$ ); panel D highlights an additional isosbestic point at $664 \mathrm{~nm}$ appearing within the spectral range 638 to $682 \mathrm{~nm}$. While the isosbestic points at 610 and $664 \mathrm{~nm}$ appear at temperatures coinciding with the formation of dsDNA from ssDNA immediately below the ss-ds DNA transition (namely ca. $60-51{ }^{\circ} \mathrm{C}$ ), the isosbestic point observed at ca. $607.5 \mathrm{~nm}$ emerges at temperatures below $51^{\circ} \mathrm{C}$. Taken together, the observation of a blue-shifted absorption feature at high temperatures, which is not consistent with the J-dimer, and the observation of distinct sets of isosbestic points over two different temperature ranges suggests that more than one aggregate population is present in the J-dimer solution. We thus assign the feature appearing at ca. $594 \mathrm{~nm}$ to a small fraction of $\mathrm{H}$-aggregate population present in the unpurified solution in addition to the larger fraction of J-dimers whose absorption maximum peaks at $666 \mathrm{~nm}$.

A)

C)
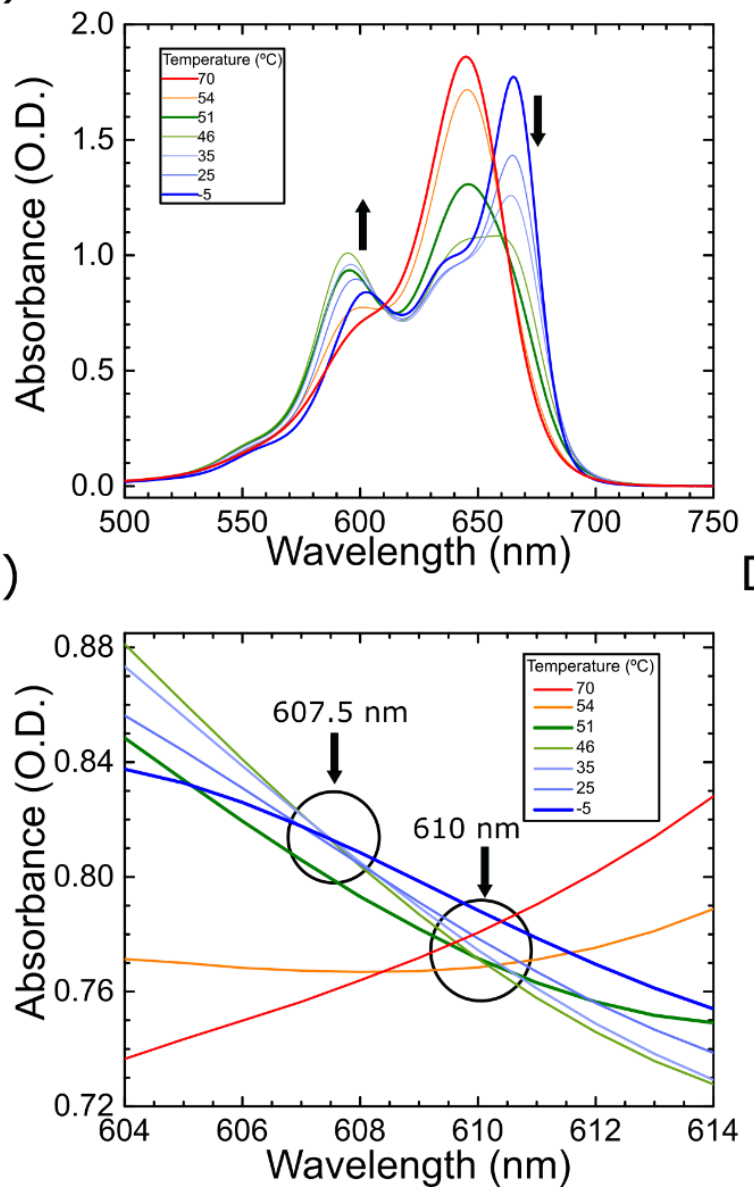

B)
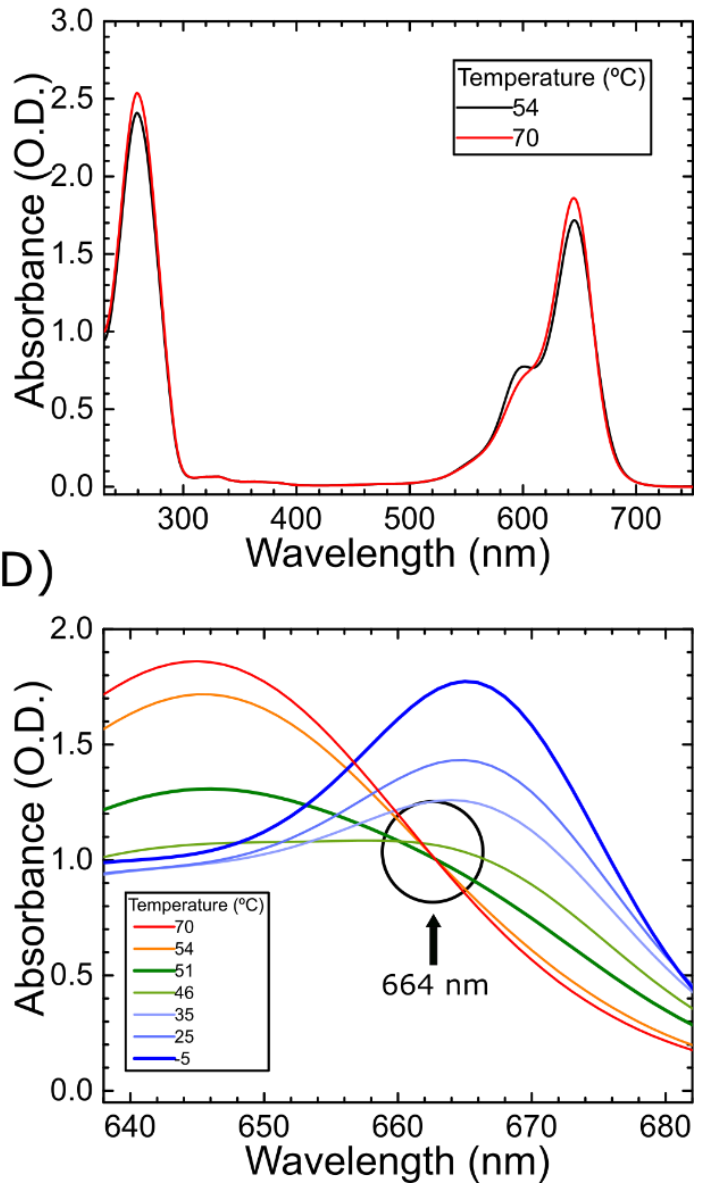
Figure S.3.2. Temperature-dependent absorption spectra of an unpurified J-dimer solution. The solution was initially heated to $70^{\circ} \mathrm{C}$, and absorption spectra were collected at temperatures ranging from $+70^{\circ} \mathrm{C}$ to $-5^{\circ} \mathrm{C}$. A) Absorption spectra over the entire temperature range, B) Absorption spectra measured at $54^{\circ} \mathrm{C}$ and $70^{\circ} \mathrm{C}$ (black and red traces, respectively). These data show that a feature at $597 \mathrm{~nm}$ is the first to emerge as the solution is cooled. The blue-shifted feature (without a corresponding red-shifted feature) is consistent with an H-aggregate packing arrangement. C) The same absorption spectra over the spectral range 604 to $614 \mathrm{~nm}$ and 0.72 to 0.88 O.D. Isosbestic points are apparent in the vicinity of ca. 610 and $607.5 \mathrm{~nm}$ over the temperature ranges $70^{\circ} \mathrm{C}$ to $51^{\circ} \mathrm{C}$ and $51^{\circ} \mathrm{C}$ to $-5^{\circ} \mathrm{C}$, respectively. D) The same absorption spectra over the spectral range from 638 to $682 \mathrm{~nm}$ and -0.05 to 2.0 O.D. An isosbestic point is apparent at $664 \mathrm{~nm}$ over the temperature range from $70^{\circ} \mathrm{C}$ to $51^{\circ} \mathrm{C}$.

Figure S.3.3 displays the corresponding temperature-dependent absorption spectra for the PAGEpurified J-dimer solution reported in the present work. As with the unpurified solution, the PAGE-purified solution was first heated to $70^{\circ} \mathrm{C}$, then cooled to $-5^{\circ} \mathrm{C}$ with absorption spectra measured at the endpoints and intermediate temperatures. Panel A displays absorption spectra of the PAGE-purified J-dimer (blue) and the unpurified J-dimer solution (red) at $70{ }^{\circ} \mathrm{C}$, normalized to the absorbance peak at $645 \mathrm{~nm}$. It is clear from the remaining peak at $594 \mathrm{~nm}$ that the PAGE-purified solution still contains some aggregate structures even at $70^{\circ} \mathrm{C}$, while the unpurified solution resembles the monomer absorption spectrum, indicating complete denaturation. Critically, the high temperature feature in the PAGE-purified solution absorbs at the same peak wavelength as the feature previously identified and attributed to an $\mathrm{H}$-aggregate population in the unpurified solution. We thus assign the feature in the PAGE-purified solution to the same H-aggregate population. Panel B shows that as the PAGE-purified solution was cooled from $70^{\circ} \mathrm{C}$, the absorption band at $594 \mathrm{~nm}$ began to grow before the $665 \mathrm{~nm}$ band. These observations are consistent with the observation above for the unpurified solution. Panel $\mathrm{C}$ shows that upon cooling to even lower temperatures, we observe a reduction of the high energy band with a concurrent rise in the low energy band and an isosbestic point at ca. $607.5 \mathrm{~nm}$, similar to what was observed for the unpurified solution (Figure S.3.2C). Such an isosbestic point indicates the conversion between two populations, possibly conversion between the H-aggregate and J-dimer populations. The high energy peak also appears to shift slightly to the red upon cooling, which was also observed for the unpurified solution, and can be taken as additional evidence for two aggregate populations with slightly different high energy absorption peaks. The full series of temperature-dependent absorption spectra are shown in panel D. 


\section{PAGE Purified J-Dimer Solution}

A)

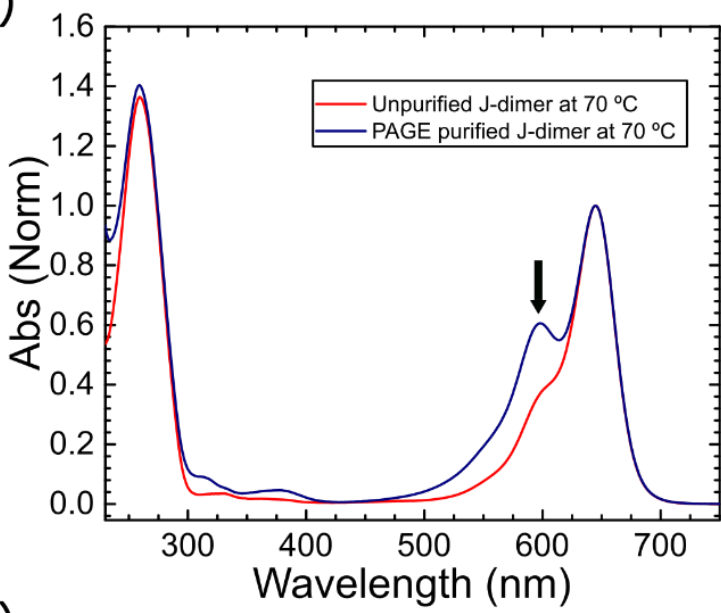

C)

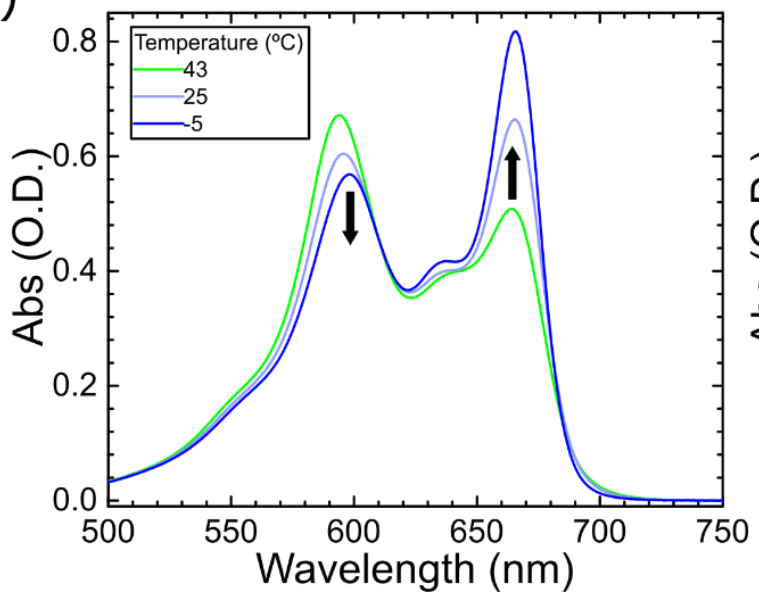

B)

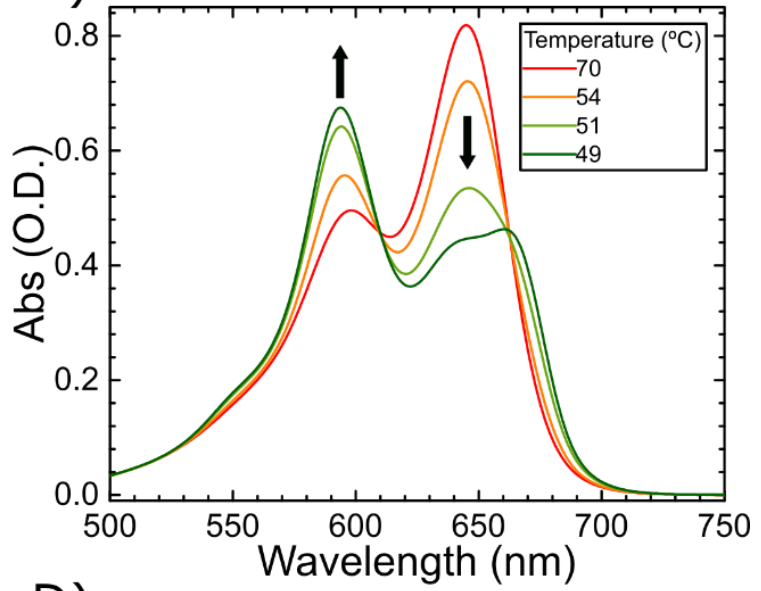

D)

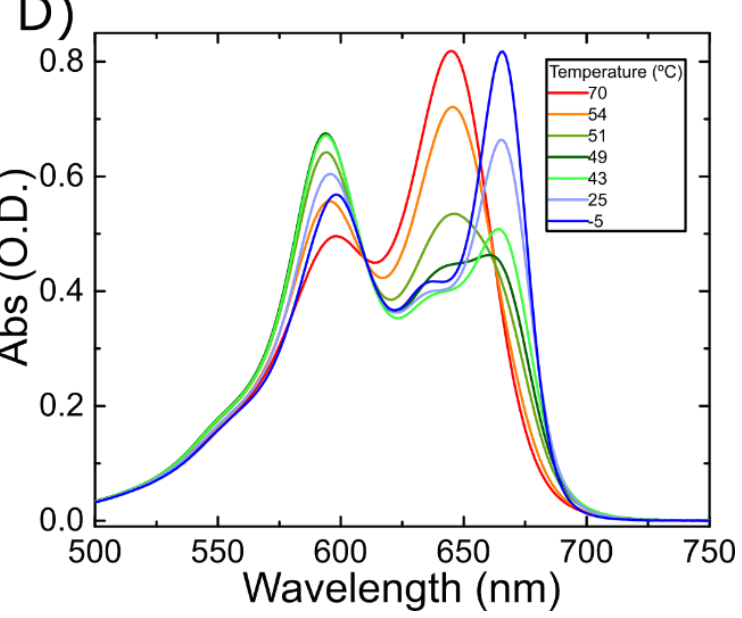

Figure S.3.3. Temperature-dependent absorption spectra for the PAGE-purified J-dimer solution reported in the present work. A) The absorption spectrum of the PAGE-purified (blue) and unpurified (red) J-dimer samples at $70^{\circ} \mathrm{C}$. The spectrum of the unpurified $J$-dimer is essentially identical to that of the monomer, while the absorption spectrum of the PAGE-purified J-dimer at $70^{\circ} \mathrm{C}$ shows obvious deviations. The peak observed at $597 \mathrm{~nm}$, which is identical to that observed in the unpurified sample at the ss-ds transition temperature, is assigned to the same H-aggregate population. B) Absorption spectra in the temperature range from $70^{\circ} \mathrm{C}$ to $49^{\circ} \mathrm{C}$. The absorption band at $665 \mathrm{~nm}$ is totally quenched at $54^{\circ} \mathrm{C}$, while a band at $597 \mathrm{~nm}$ is still apparent even at 70 ${ }^{\circ} \mathrm{C}$. C) Absorption spectra at 43, 25, and $-5^{\circ} \mathrm{C}$. These data show that the absorbance at $594 \mathrm{~nm}$ decreases and the absorbance at $665 \mathrm{~nm}$ increases within this temperature range. D) Absorption spectra collected over the entire temperature range from $70^{\circ} \mathrm{C}$ to $-5^{\circ} \mathrm{C}$.

Taken together, these results indicate that, in addition to the primary population of duplex DNA Jdimer aggregates, a minor population of $\mathrm{H}$-aggregates is present in the PAGE-purified J-dimer solution reported and studied in the present work. Given that the absorption spectrum of the unpurified J-dimer solution is not appreciably different from the absorption spectrum of the J-dimer solution purified according to the procedure of Cannon et al., and given that only a small amount of the H-aggregate population was observed upon hybridizing the DNA strands in the unpurified J-dimer solution, we further consider the absorption spectrum of the unpurified J-dimer solution as the best representation of the 'pure' J-dimer absorption spectrum. 


\section{Supporting Information S.4:}

\section{$4 \quad$ Monomer Photophysics}

In this section, we show that the photophysics of monomers $\mathrm{A}$ and $\mathrm{B}$ (Cy5-A and Cy5-B, respectively; Supporting Information 1.2: DNA Sequences) are essentially equivalent. Figure S 4.1 displays the steady-state absorption and fluorescence spectra of monomers A and B. The spectra are nearly indistinguishable.

Absorption

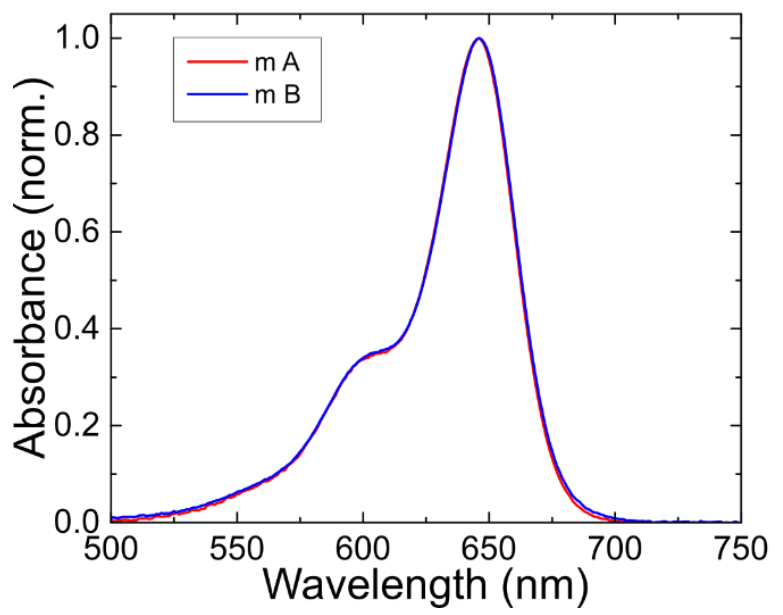

Fluorescence

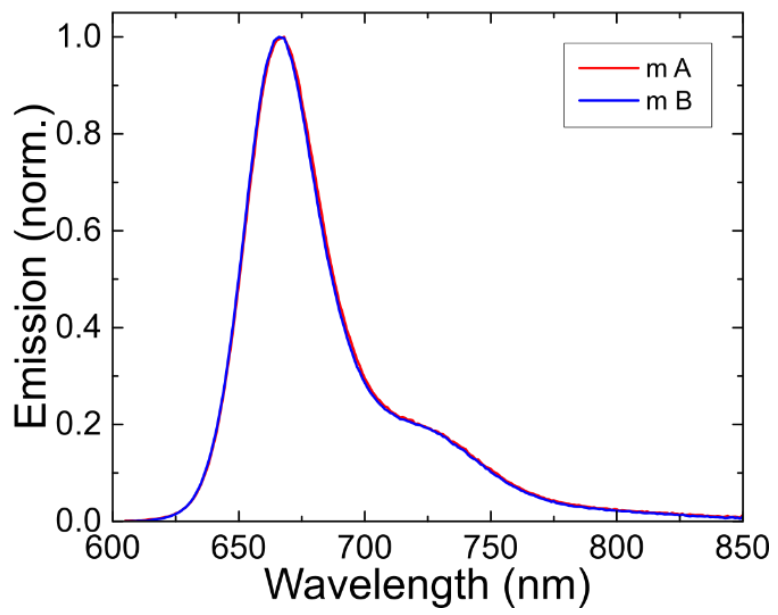

Figure S 4.1. Steady-state absorption (left) and fluorescence (right) spectra of monomer A (red traces) and monomer B (blue traces) are virtually indistinguishable.

The fluorescence quantum yields of monomers A and B have also been measured and found to be 0.29 and 0.33 , respectively. The slightly larger fluorescence quantum yield for monomer B is consistent with its slightly longer lifetime (and presumably reduced nonradiative decay rate) relative to monomer A as observed via TCSPC measurements (Figure S 4.2). Specifically, the lifetimes of monomers A and B determined from TCSPC were ca. 1.3 and $1.5 \mathrm{~ns}$, respectively.
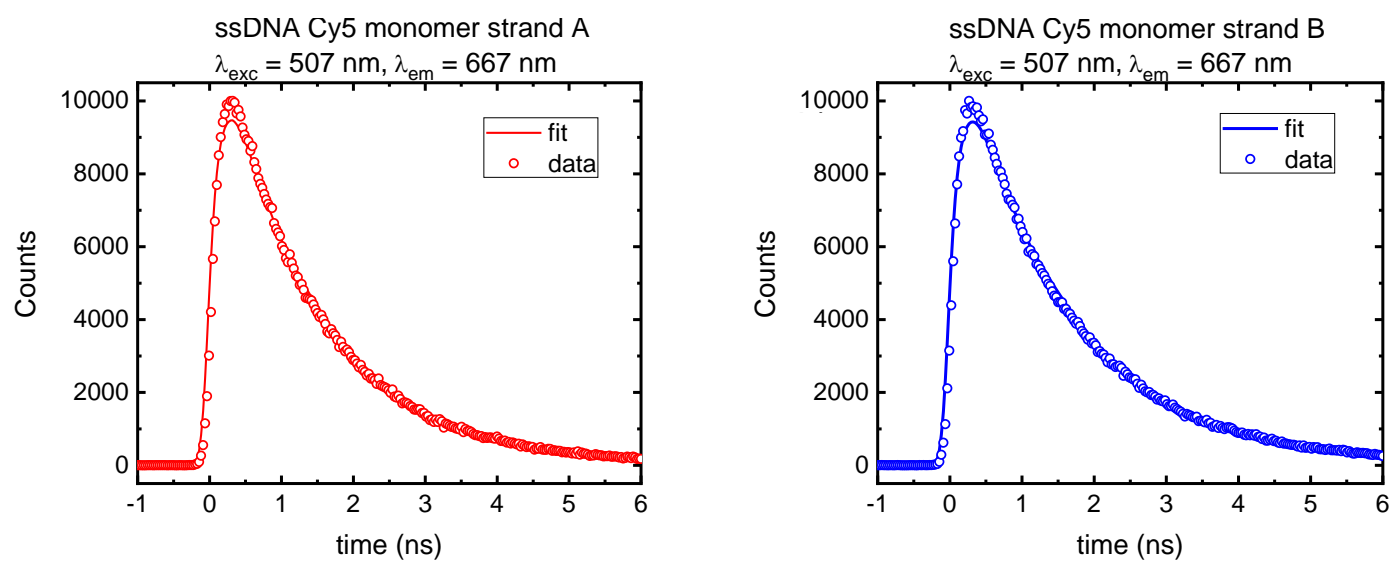
Figure S 4.2. Time-correlated single photon counting measurements of monomer A (left, red) and B (right, blue). Open circles are the data and lines are fits.

Given these very minor variations in the steady-state and time-resolved optical properties of monomers $\mathrm{A}$ and $\mathrm{B}$, for the remainder of the manuscript (including this Supporting Information) all references to the "monomer" refer specifically to the "monomer A" or "Cy5-A" strand (Supporting Information 1.2: DNA Sequences), unless otherwise noted. 


\section{Supporting Information S.5:}

\section{Fluorescence Excitation Spectroscopy Establishes the}

\section{Multicomponent Nature of Aggregate Solutions}

By monitoring the fluorescence intensity at a single wavelength while simultaneously scanning the excitation wavelength, the absorption spectrum of the emissive species can be mapped out. ${ }^{3}$ In the case of a solution containing multiple fluorescent species with distinct absorption and emission spectral profiles, one can expect such a fluorescence excitation measurement to return a convolution of the absorption spectra of the individual species with the relative amplitude of each species depending on its relative concentration, molar absorption coefficient, and fluorescence quantum yield.

Figure S 5.1 displays the fluorescence excitation spectrum and fluorescence emission spectrum of the PAGE-purified J-dimer sample. Included for comparison are the UV-Vis absorption spectra of the Jdimer and monomer. The overall shape of the excitation spectrum of the PAGE-purified J-dimer solution is essentially equivalent to the absorption spectrum of the monomer. The results of fluorescence excitation measurements on the PAGE-purified H-tetramer solution (Figure S 5.2) are similar to that of the PAGEpurified J-dimer solution: both excitation spectra look essentially equivalent to the monomer absorption spectrum and lack the strong, blue shifted peak characteristic of the aggregated species (ca. $600 \mathrm{~nm}$ for the J-dimer and ca. $565 \mathrm{~nm}$ for the H-tetramer). Thus, while the monomers present in the PAGE-purified aggregate solutions contribute negligibly to the absorption spectra of PAGE-purified aggregate samples, they are responsible for essentially all of the observed fluorescence emission.

PAGE Purified J-dimer

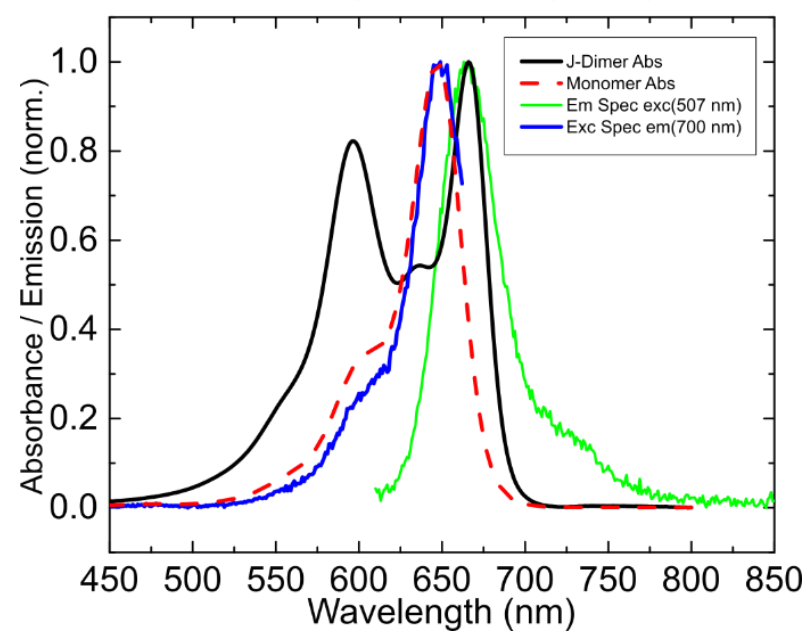

Figure S 5.1. Steady-state spectra of the PAGE purified J-dimer solution. The absorption spectrum of the J-dimer is shown in gray. TheJ-dimer emission spectrum upon excitation at $507 \mathrm{~nm}$ is shown in green. The fluorescence excitation spectrum for PAGE purified J-dimer solution, monitored at $700 \mathrm{~nm}$, is given in dark blue. The absorption spectra of the single strand attached Cy5 monomer is given in the red dashed line for comparison. 


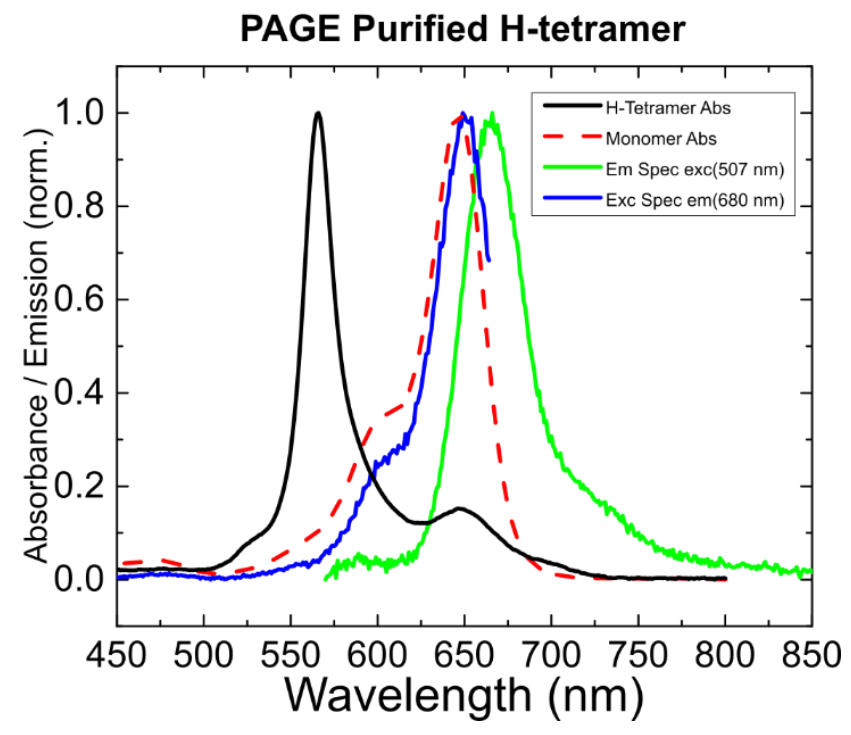

Figure S 5.2. Steady-state spectra of the PAGE purified H-tetramer solution. The absorption spectrum of the H-tetramer is plotted in black. The H-tetramer emission spectrum upon excitation at $507 \mathrm{~nm}$ is shown in green. The fluorescence excitation spectrum for PAGE purified H-tetramer solution, monitored at $680 \mathrm{~nm}$, is given in dark blue. The absorption spectra of the single strand attached Cy5 monomer is given in the red dashed line for comparison. 


\section{Supporting Information S.6:}

\section{Extraction of the "Pure" J-dimer Emission Spectrum}

\subsection{Theory}

In this section, we report a procedure for extracting the 'pure' emission spectrum of the J-dimer aggregate from the emission spectrum measured for the multicomponent $\mathrm{J}$-dimer solution. The extraction procedure was motivated in large part because of the overwhelming contribution to the fluorescence emission arising from the monomer subcomponent, present even in the PAGE-purified J-dimer solution (see Supporting Information S.5).

The extraction procedure is based on the idea that the fluorescence quantum yield collected at a single wavelength for a solution containing multiple fluorescent species is separable into the product of the relative absorbance and fluorescence quantum yield associated with each constituent species. That is:

$$
\Phi_{F, T o t}(\lambda)=\sum_{i=1}^{n}\left(\frac{A_{i}(\lambda)}{A_{T o t}(\lambda)}\right) \Phi_{F, i} \quad \text { (Equation S6.1) }
$$

where $\Phi_{F, T o t}$ and $A_{T o t}$ are the total fluorescence quantum yield (FQY) and absorbance of the solution, respectively, and $\Phi_{F, i}$ and $A_{i}$ are the FQY and absorbance of a particular subcomponent or species. For the present case of the J-dimer solution, there is evidence for three constituent populations. The primary species, the J-like dimers, are evident from the absorption spectrum (Figure $\mathbf{2}$ in main text). Two additional species are present in the solution. Fluorescence excitation measurements indicate that the J-dimer solutions contain an additional Cy5 monomer species (Supporting Information S.5), and temperature-dependent $\mathrm{UV} / \mathrm{V}$ is absorption measurements indicate that a small population of $\mathrm{H}$-like aggregates is also present in the solution (Supporting Information S.3). Thus, we arrive at the following expression for the FQY of the three-species solution:

$$
\Phi_{F, T o t}(\lambda)=\left(\frac{A_{m}(\lambda)}{A_{T o t}(\lambda)}\right) \Phi_{F, m}+\left(\frac{A_{J}(\lambda)}{A_{T o t}(\lambda)}\right) \Phi_{F, J}+\left(\frac{A_{H}(\lambda)}{A_{T o t}(\lambda)}\right) \Phi_{F, H}
$$

(Equation S6.2)

where $A_{m}, A_{J}$, and $A_{H}$ are the absorbance of $\mathrm{Cy} 5$ monomers, J-dimers, and $\mathrm{H}$-aggregate species, respectively, and $\Phi_{F, m}, \Phi_{F, J}$, and $\Phi_{F, H}$ are the FQY of the monomer, J-dimer, and $\mathrm{H}$-aggregate species, respectively. The FQY of each species is also assumed to be independent of the excitation wavelength (i.e., Vavilov's rule ${ }^{3}$ ). The terms on the right sides of Equation S6.1 and S6.2 represent the individual contributions of the three components to the total emission of the solution when excited at a particular wavelength. Notice that, although we have assumed that the FQY of the individual species is independent of excitation wavelength, the FQY of the J-dimer solution remains a function of excitation wavelength. This is because the absorbance of the different species remains a function of excitation wavelength.

We next make the assumption that the H-aggregate species is non-fluorescent over the spectral range of interest and that it only contributes to the absorption spectrum of the J-dimer solution; thus, we assume only the J-dimer and monomer components contribute to the measured emission. This assumption is reasonable because H-aggregates are known to exhibit substantially reduced radiative rates $^{4}$ which, in conjunction with rapid ground state recovery (see main text and Supporting Information S.12), results in a 
greatly suppressed quantum yield of fluorescence emission. Since we assume that the H-aggregate component is non-emissive, the third term on the right side of equation S6.2 becomes 0. Additionally, since the total absorbance of the solution, $A_{T o t}$, is equal to the sum of the absorbance of the three populations, the absorbance of the pure J-dimer component can be expressed as $A_{J}=A_{T o t}-A_{m}-A_{H}$. Equation S6.2 becomes:

$$
\Phi_{F, T o t}(\lambda)=\left(\frac{A_{m}(\lambda)}{A_{T o t}(\lambda)}\right) \Phi_{F, m}+\left(\frac{A_{T o t}(\lambda)-A_{m}(\lambda)-A_{H}(\lambda)}{A_{T o t}(\lambda)}\right) \Phi_{F, J}
$$

(Equation S6.3)

The extraction procedure requires that we solve for $A_{m}$ by substituting values for all other quantities in equation S6.3. This can be done via a combination of measurement and approximation. The directly measurable quantities are $\Phi_{F, T o t}, \Phi_{F, m}$, and $A_{T o t}$, while the approximated parameters are $\Phi_{F, J}$ and $A_{H}$.

\subsection{Procedure}

The procedure to extract the 'pure' J-dimer emission spectrum is as follows: (i) collect absorption and fluorescence emission spectra of the J-dimer solution, (ii) collect absorption and fluorescence emission spectra of a highly dilute monomer solution with equivalent spectrometer settings, (iii) solve equation S6.3 to determine the absorbance that the monomer contributes to the J-dimer solution absorption spectrum, and (iv) scale the dilute monomer solution emission spectrumaccordingly and subtract its contribution from the J-dimer solution emission spectrum, leaving only the 'pure' emission spectrum of the J-dimer species.

\subsubsection{UV-Vis and Fluorescence Spectroscopy}

Samples of Monomer B and the J-dimer solution were prepared according to Section S2.1. The Jdimer solution was subjected to PAGE purification as described in Section S1.4. The samples were prepared at concentrations such that the peak emission counts, as measured on the spectrofluorometer without changing settings, were similar for the two solutions. Figure S 6.1 shows the absorption and corresponding emission spectra of the two solutions. 

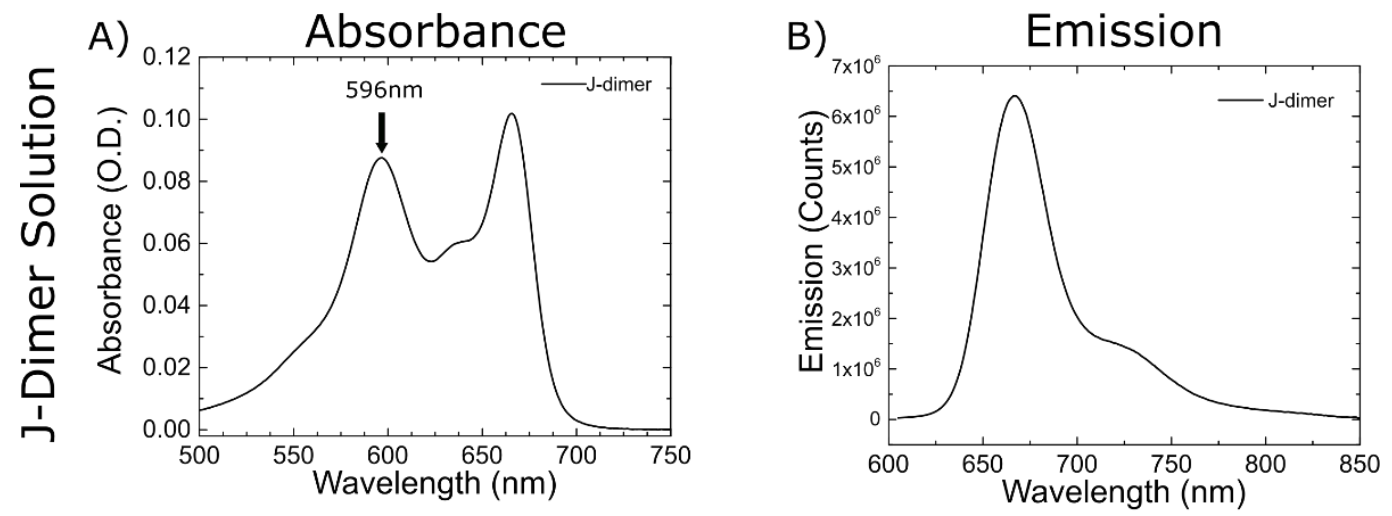

C)
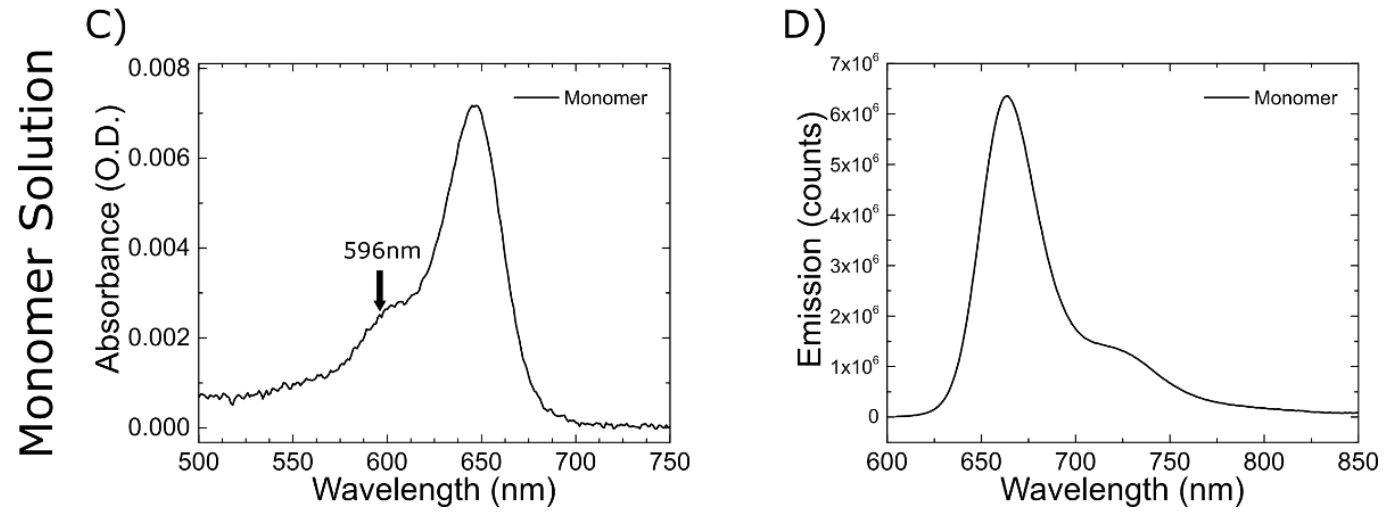

Figure S 6.1. A) Absorbance and B) corresponding fluorescence emission spectrum of the PAGE purified J-dimer solution. C) Absorbance and D) emission spectrum of the monomer solution. Emission spectra were collected while exciting at $596 \mathrm{~nm}$.

\subsubsection{Fluorescencequantum yields}

Relative FQY measurements were performed on the Monomer B and J-dimer solutions. The fluorescence quantum yield of Monomer B, $\Phi_{F, m}$, was measured to be 0.33 (Supporting Information S.4). As noted above, we assume that the FQY of each species is independent of the excitation wavelength. As shown in Eqn. S6.1, however, a multicomponent solution can still remain dependent on excitation wavelength. Accordingly, the FQY of the J-dimer solution, $\Phi_{F, T o t}(\lambda)$, was measured at a specific wavelength; when excited at $596 \mathrm{~nm}$, the FQY of the J-dimer solution was determined to be 0.010 .

In the present case, the fluorescence quantum yield of the 'pure' J-dimer, $\Phi_{F, J}$, cannot be measured directly, and was therefore estimated via the following general expression:

$$
\Phi_{F, i}=\frac{k_{r, i}}{k_{r, i}+k_{n r, i}}=\frac{k_{r, i}}{k_{o b s, i}} \quad \text { (Equation S6.4) }
$$

where $\Phi_{F, i}$ is the FQY of a specific component and $k_{r, i}, k_{n r, i}$, and $k_{o b s, i}$ are the radiative, nonradiative, and observed decay rates of the specific component. For the observed decay rate of the J-dimer, $k_{o b s, J}$, transient absorption spectroscopy yielded a value of $93 \mathrm{~ns}^{-1}$ (see main text). While $k_{o b s, J}$ can be measured directly, the value of $k_{r, J}$ cannot. The only way the value of $k_{r, J}$ can be derived, without knowledge of $\Phi_{F, J}$, is via the extinction spectrum, which is exceptionally difficult if at all possible (as is alluded to in the main text). Thus, the value of $k_{r, J}$ was estimated in the following manner. First, the radiative rate of the Cy5 monomer, $k_{r, m}$, was calculated by substituting the FQY of Monomer B $\left(\Phi_{F, m}=0.33\right)$ and monomer relaxation rate $\left(k_{o b s, m}=0.66 \mathrm{~ns}^{-1}\right)$ into Equation S6.4 and solving for $k_{r, m}$. The radiative rate for the monomer was found to 
be $0.22 \mathrm{~ns}^{-1}$. Due to the nature of superradiance, a J-dimer, which by definition comprises two molecules, can have a radiative rate ranging from that of the monomer to twice that of the monomer. ${ }^{5,6}$ Based on the extinction spectra reported in ref. 1 , where the maximum extinction of the $\mathrm{J}$-dimer is 1.5 times that of the monomer (i.e., $\varepsilon_{m}(\max ) \sim 200,000 \mathrm{M}^{-1} \mathrm{~cm}^{-1}$ and $\left.\varepsilon_{J}(\max ) \sim 300,000 \mathrm{M}^{-1} \mathrm{~cm}^{-1}\right)$, we estimate the radiative rate of the J-dimer to be 1.5 times that of the monomer, or $0.33 \mathrm{~ns}^{-1}$. Thus, we have a measured value for $k_{o b s, J}$ $=93 \mathrm{~ns}^{-1}$ and an estimated value for $k_{r, J}=0.33 \mathrm{~ns}^{-1}$. Substituting these values into Equation S6.4, a value of $4 \times 10^{-3}$ was obtained for $\Phi_{F, J}$.

\subsubsection{Estimating the H-aggregate absorption contribution}

In order to extract the 'pure' J-dimer fluorescence emission spectrum, the next step of the procedure is to solve equation S.6.3 for $A_{m}$ (at $596 \mathrm{~nm}$ ), scale the emission spectrum of the monomer (Figure S6.1, bottom right) by the ratio $A_{m} / A_{m, s o l}$ ' , where $A_{m, s o l ' n}$ is the absorbance of the monomer solution (Figure S6.1, bottom left), then subtract the scaled monomer emission spectrum from the emission spectrum collected for the J-dimer solution (Figure S6.1, top right). The only value listed in equation S6.3 that has yet to be either measured or estimated (aside from $A_{m}$ ) is $A_{H}\left(\right.$ at $596 \mathrm{~nm}$ ). With all other values set, the choice of $A_{H}$ determines $A_{m}$ and the associated scaling factor. Without an obvious way to measure or estimate $A_{H}$, the extraction was performed for four values of $A_{H}$, i.e., $A_{H}=0,30,60$, and $90 \%$ of the total absorbance of the solution (Table S6.1). The fluorescence emission spectra extracted according to this procedure are presented in Figure S6.2.

Table S6.1. The absorbance of $A_{H}$ expressed as percent of the J-dimer absorption at $596 \mathrm{~nm}$ (first column) and optical density (second column), and the resulting values of $A_{m}$ (third column). Note that $A_{\text {Tot }}=0.0875$. Additional digits beyond those which are significant are included for illustrative purposes.

\begin{tabular}{|c|c|c|}
\hline $\boldsymbol{A}_{\boldsymbol{H}} / \boldsymbol{A}_{\text {Tot }}(\boldsymbol{\%})$ & $\boldsymbol{A}_{\boldsymbol{H}}$ & $\boldsymbol{A}_{\boldsymbol{m}}$ \\
\hline $0 \%$ & 0 & 0.0017 \\
\hline $30 \%$ & 0.0262 & 0.0020 \\
\hline $60 \%$ & & \\
\hline & 0.0525 & 0.0023 \\
\hline $90 \%$ & & \\
\hline & 0.0787 & 0.0026 \\
\hline
\end{tabular}




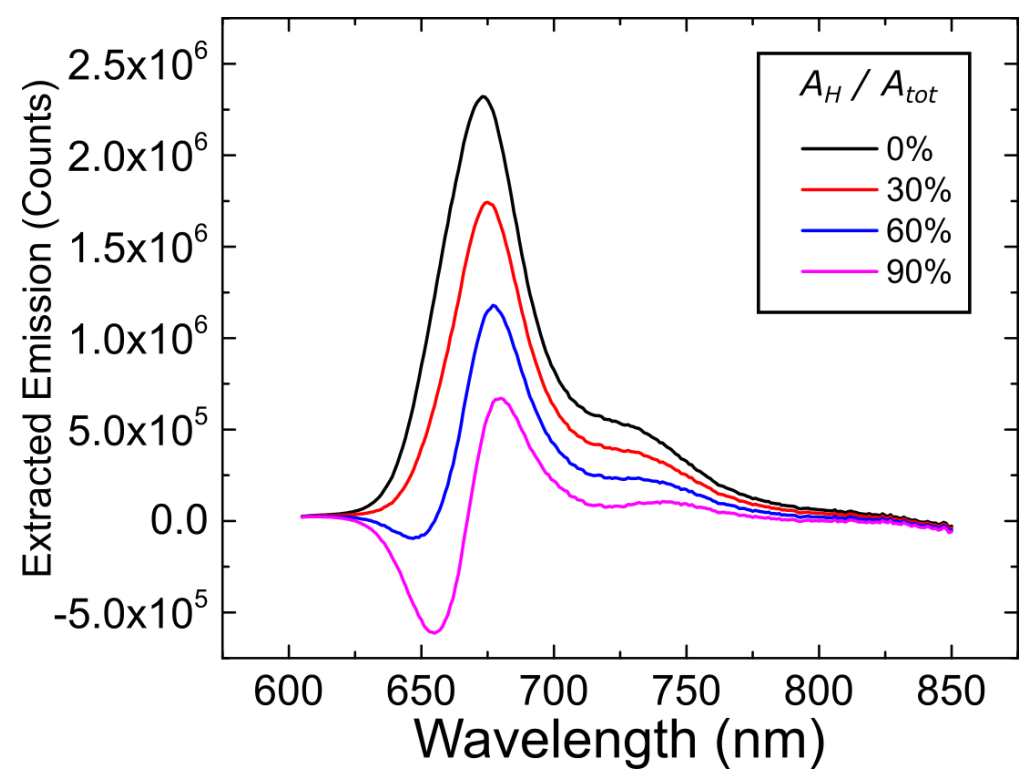

Figure S 6.2. Fluorescence emission spectra derived by subtracting the monomer emission spectrum scaled according to different values of $A_{H}$ at $596 \mathrm{~nm}$.

Figure S6.2 shows that, for large values of $A_{H}$, the spectra derived from the extraction procedure exhibit negative intensity at short wavelengths. Since negative emission intensity in a fluorescence emission measurement is not physically reasonable, we exclude the possibility that $A_{H}$ is greater than $60 \%$ of $A_{t o t}$. This result is not entirely unexpected - the H-aggregate component, identified in Supporting Information $\mathrm{S} .3$, is a minor population. Figure S6.2 also shows that the width of the extracted J-dimer fluorescence emission origin band also varies with $A_{H}$.

Having identified that the width of the extracted $\mathrm{J}$-dimer fluorescence emission origin band varies with $A_{H}$, we used this observable as another way to constrain $A_{H}$ (and, by extension, $A_{m}$ ). Namely, we assume that the absorption and emission spectra of the 'pure' J-dimer exhibit mirror symmetry, ${ }^{7}$ such that the linewidths in absorption and emission should be similar, if not the same. The full width at half maximum (FWHM), approximated as twice the half width at half maximum (HWHM), of the vibronic origin band of the extracted J-dimer fluorescence emission spectrum is ca. $650 \mathrm{~cm}^{-1}$. A maximum $A_{H}$ value, $49 \%$ of $A_{\text {tot }}$ was determined to return the narrowest 'pure' J-dimer emission spectrum while maintaining positive fluorescence emission intensities. The resulting extracted spectrum is shown in Figure S 6.3. 


\section{Extracted Pure J-dimer Emission Spectrum}

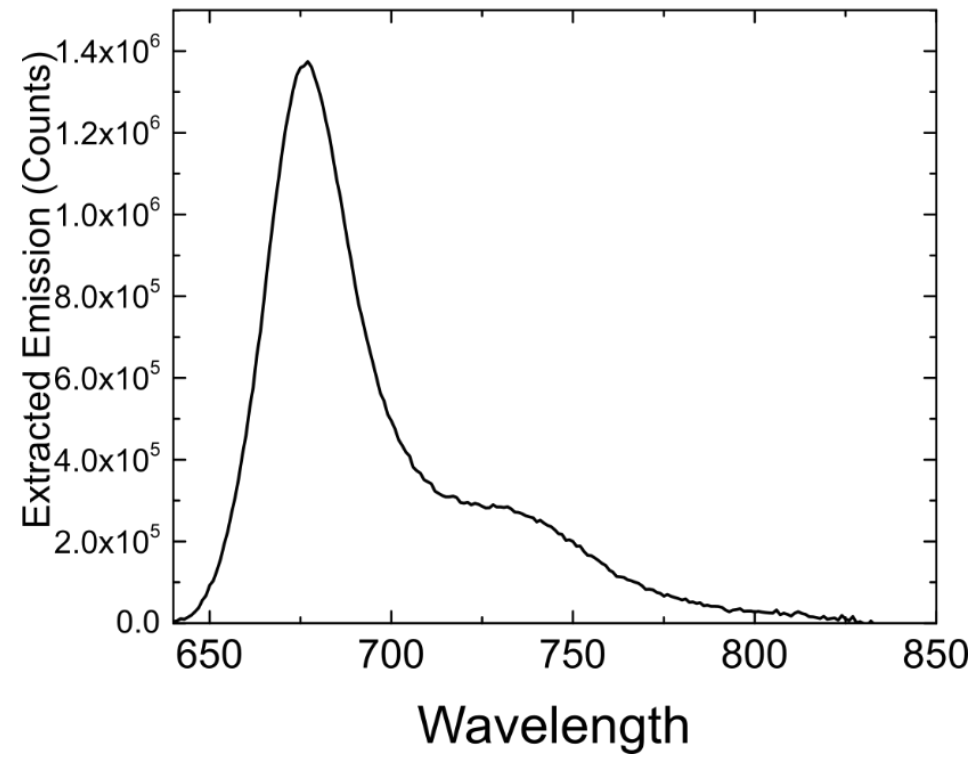

Figure S 6.3. Extracted J-dimer emission spectrum used in main text, Figure 2.

Figure S 6.3 shows that the origin band of the 'pure' J-dimer emission peaks at $677 \mathrm{~nm}$. When considering that the origin band of the J-dimer absorption spectrum peaks at $666 \mathrm{~nm}$ (Figure 2), a Stokes shift of ca. $240 \mathrm{~cm}^{-1}$ is determined. The Stokes shift determined for the 'pure' J-dimer is much less than the value of $440 \mathrm{~cm}^{-1}$ determined for the monomer. Furthermore, the FWHM of the 'pure' J-dimer emission spectrum is, according to the extraction, estimated to be ca. $650 \mathrm{~cm}^{-1}$. This value is similar to the J-dimer absorption FWHM of $550 \mathrm{~cm}^{-1}$, and narrower than the FWHM of the origin band of the monomer emission spectrum, which is $790 \mathrm{~cm}^{-1}$. The former observation is consistent with our assumption of mirror symmetry; the latter observation is consistent with our understanding (and assumptions) that the molecules composing the J-dimer can be treated in the limit of exciton delocalization (i.e., $N>1$ ), which leads to spectral narrowing of the emission. 


\section{Supporting Information S.7:}

\section{$7 \quad$ H-tetramer Solution Emission}

A number of factors complicated applying the same technique used for extracting the pure J-dimer spectrum (Supporting Information S.6) to extract the pure H-tetramer emission spectrum. Specifically, the presence of both monomer and J-dimer components in the H-tetramer solution, as well as the exceedingly low fluorescence quantum yield of the entire solution $\left(\Phi_{\mathrm{H}, \mathrm{Tot}}=3 \times 10^{-4}\right.$; see main text), made a quantitative extraction impossible. There is evidence, however, that the H-tetramer emits in the vicinity of 760 to 850 $\mathrm{nm}$ as determined by comparison of the normalized emission spectra for all samples. Figure $\mathrm{S} 7.1$ and Figure S 7.2 show overlays of the normalized emission spectra for the pure monomer, J-dimer solution, and H-tetramer solution. Figure S 7.2 is a magnification of the boxed area (long wavelength spectral tails) in Figure S 7.1. The H-tetramer solution emission spectrum shows a small, but significant fluorescence emission signal at long wavelengths that is not observed in either of the other solutions. These results are consistent with a strongly redshifted and featureless molecular 'excimer' emission observed in aggregates of other structurally-related cyanine dyes..$^{8,9}$

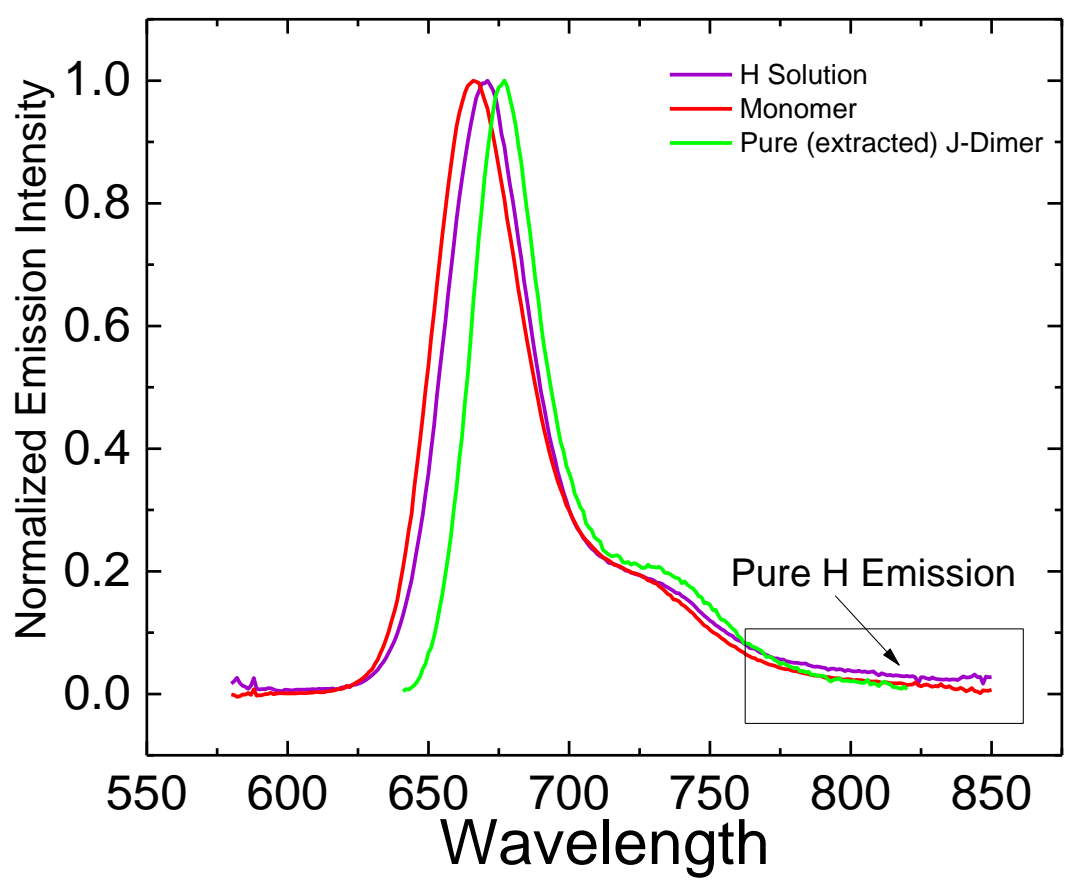

Figure S 7.1. An overlay of the normalized emission spectra of the H-tetramer solution (violet), monomer (red), and J-dimer (green). 


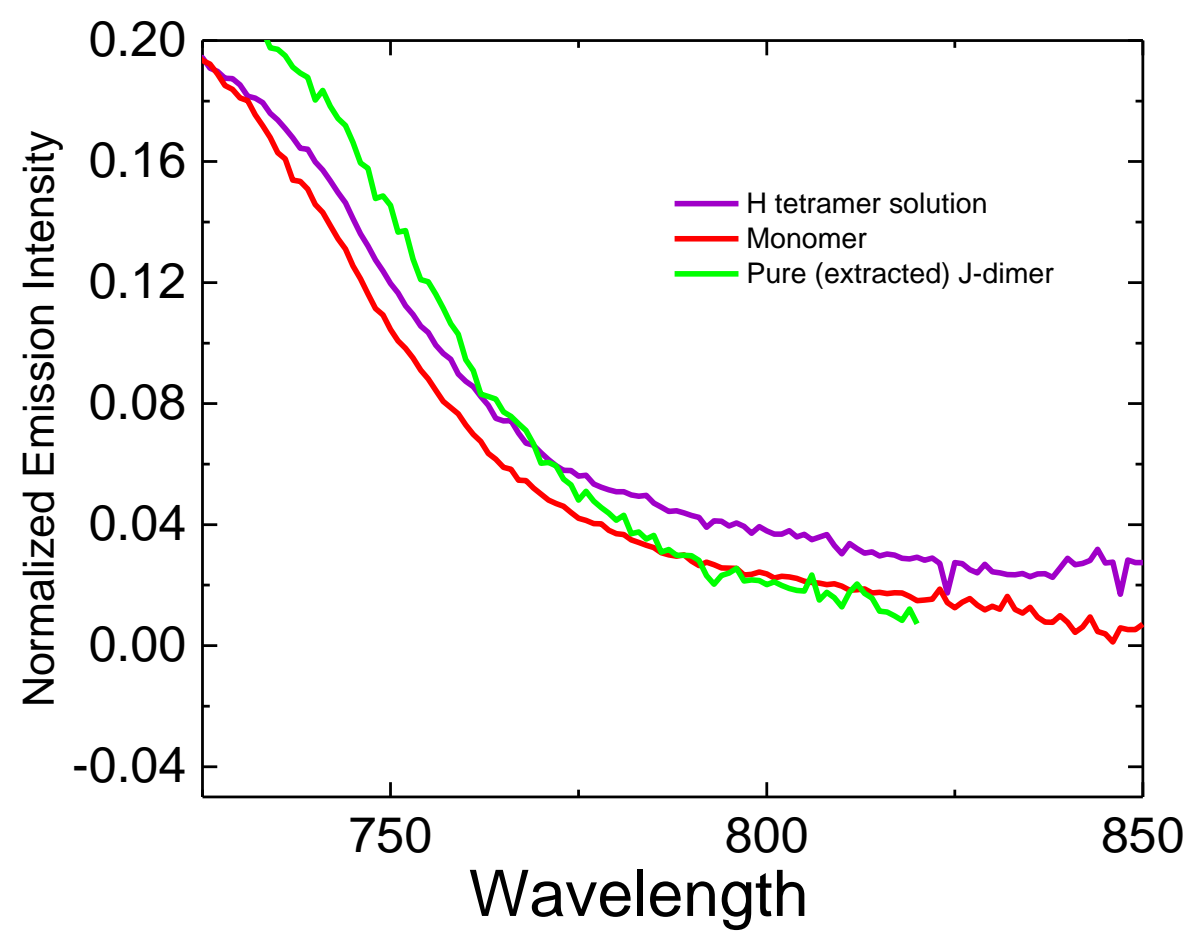

Figure S 7.2. Enlarged view of the boxed area in Figure $\boldsymbol{S}$ 7.1. The H-tetramer solution (violet) shows increased fluorescence emission relative to all other samples starting at ca. $760 \mathrm{~nm}$. 


\section{Supporting Information S.8:}

8 Mathematical and Physical Justification of Biexponential Fits for Aggregate Solution Time-Resolved Fluorescence

The fluorescence decay for both aggregate samples as measured by time-correlated single photon counting (TCSPC) was characterized by an initial, rapid decay followed by a much slower decay to baseline. To determine the number of exponential functions necessary to accurately model these data, single exponential and biexponential functions were used to fit both the J-dimer and H-tetramer TCSPC data and the relative goodness of the fits was compared.

The single exponential and biexponential fits and their residuals are displayed for the J-dimer and H-tetramer solutions in Figure S 8.1 and Figure S 8.2, respectively. In the case of the biexponential fit for the H-tetramer solution, where the long-time component is very low in amplitude, the longer time constant was fixed at $1.3 \mathrm{~ns}$, corresponding to the measured lifetime of the Cy5 monomer.

\section{Single Exponential}

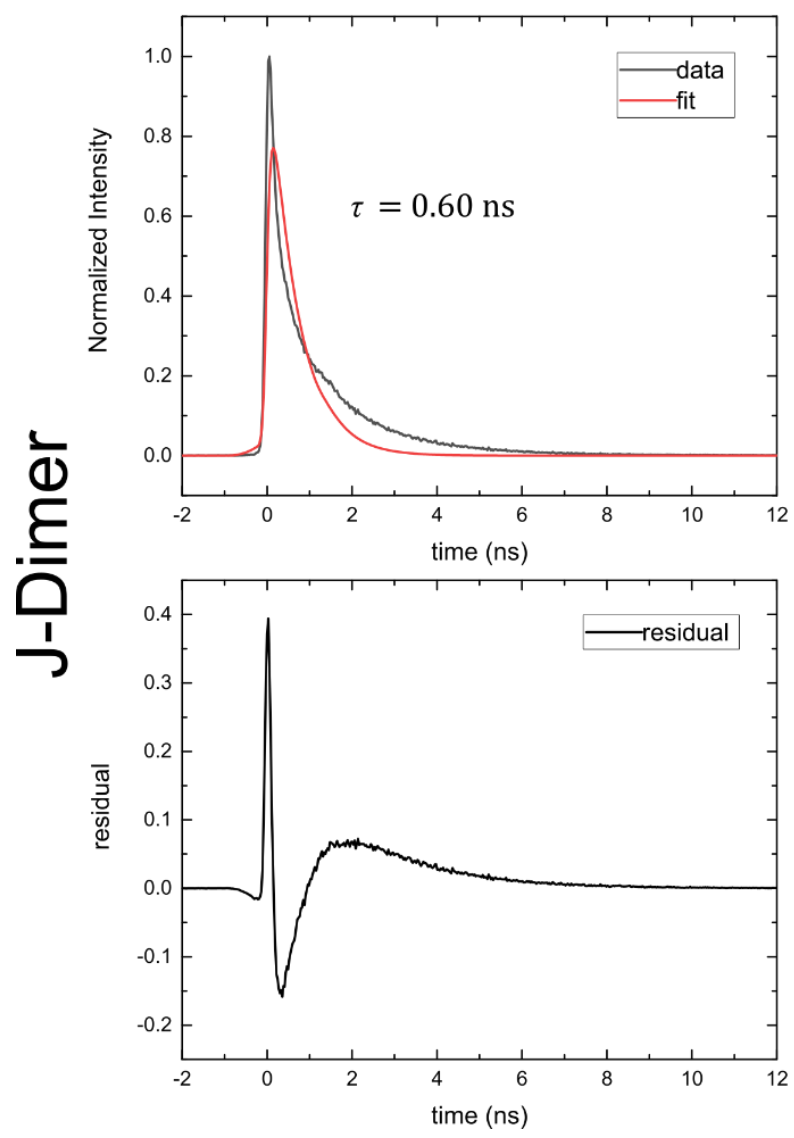

Biexponential
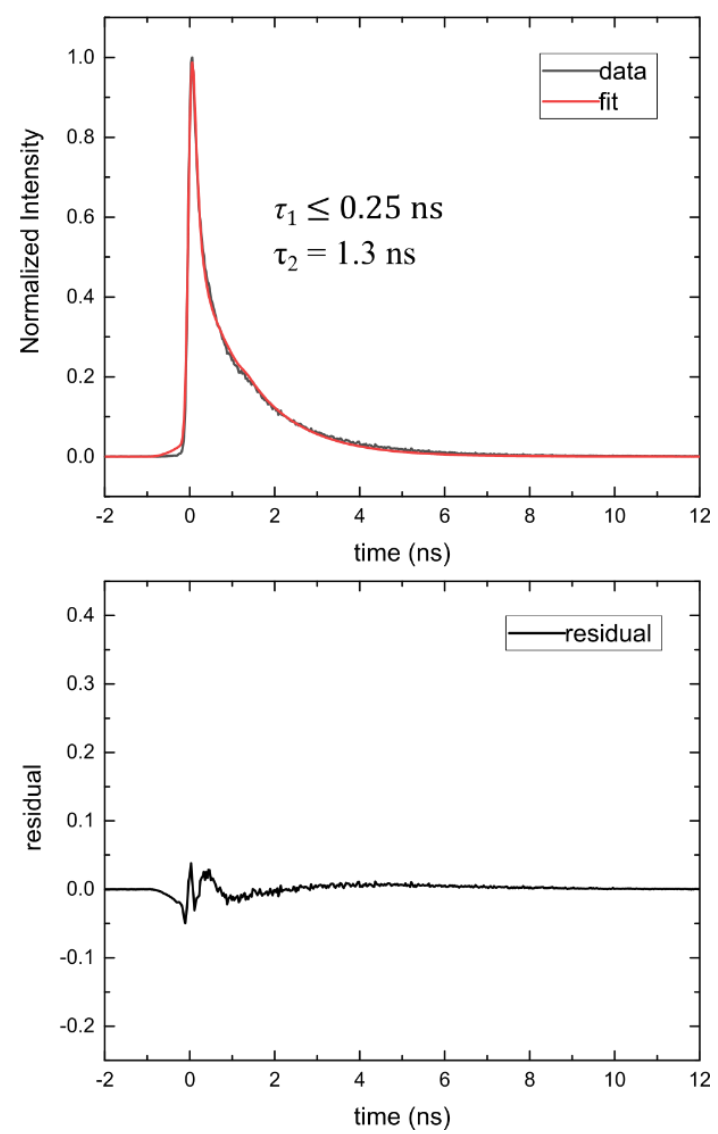

Figure S 8.1. Data (black traces) and fits (red traces) resulting from a single (left) and biexponential (right) fit to model the TCSPC decays of the J-dimer solution. The better residual and smaller sum of square of residuals indicates that a biexponential fit better models the data. 


\section{Single Exponential}
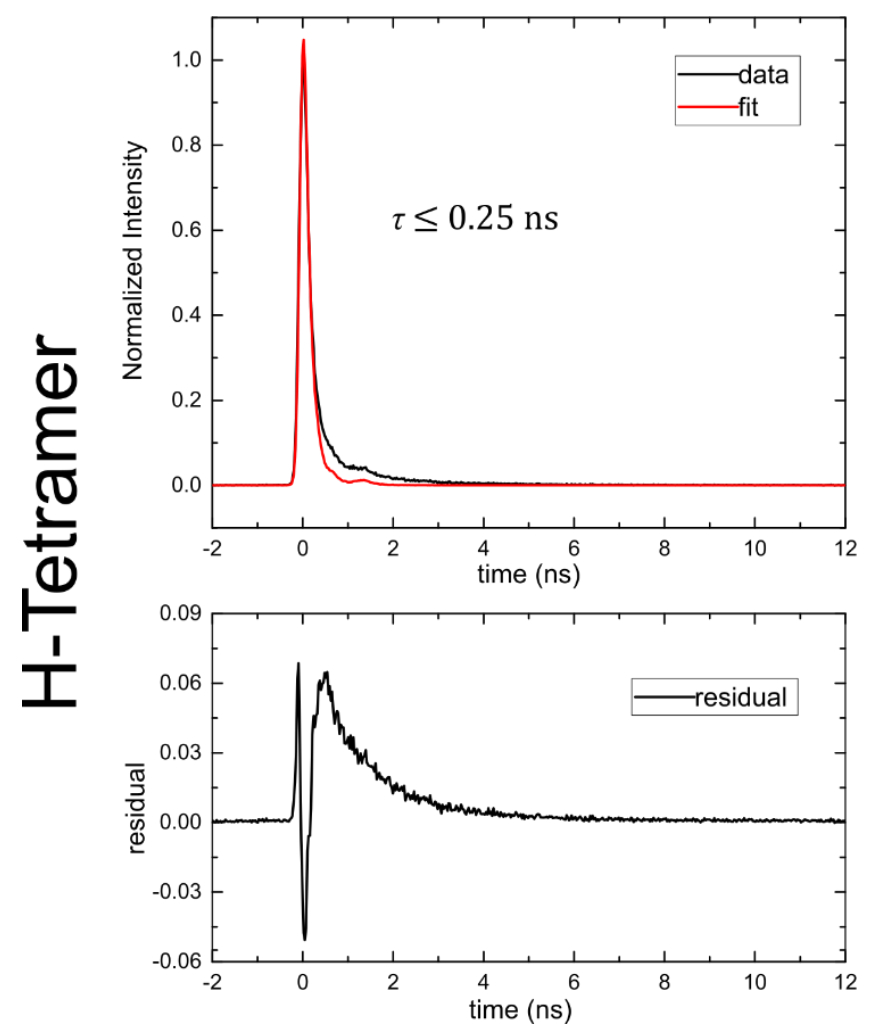

Biexponential
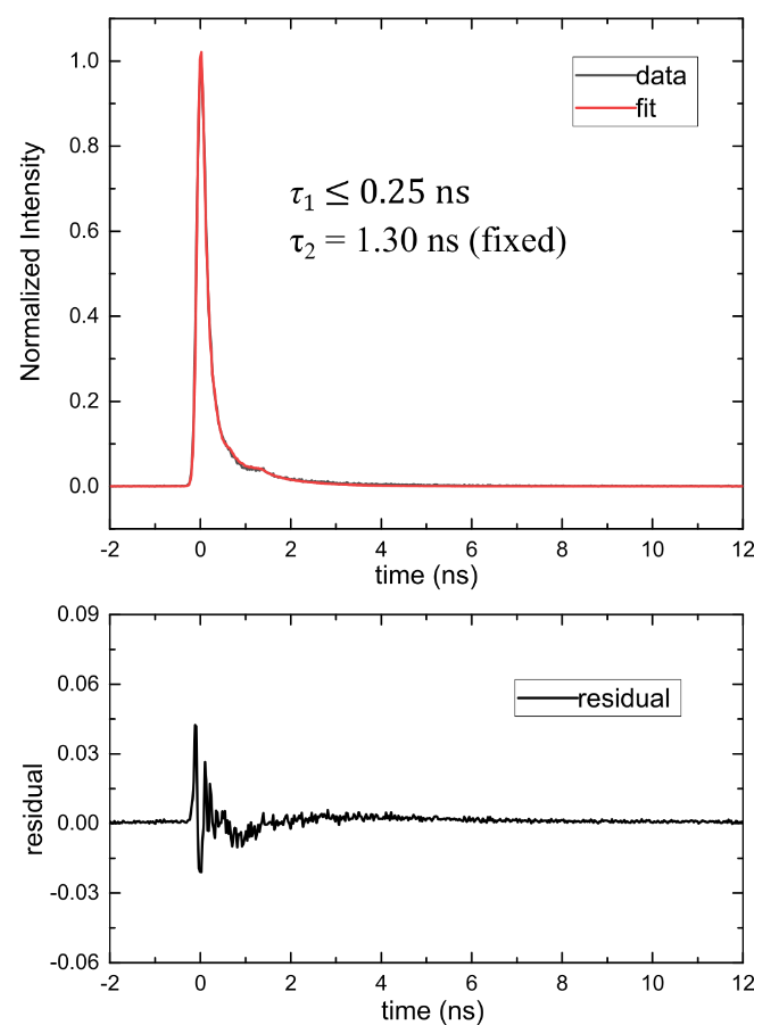

Figure S 8.2. Data (black traces) and fits (red traces) resulting from a single (left) and biexponential (right) fit to model the TCSPC decays of the H-tetramer solution. The better residual and smaller sum of square of residuals indicates that a biexponential fit better models the data.

The best fit single exponential decays for both construct solutions fail to capture the full character of the fluorescence decay as evidenced by the poor agreement between the data and the fits and the structure apparent in their respective residuals (Figure S 8.1 and Figure S 8.2, left panels). The inclusion of an additional exponential term to the fitting functions (Figure $\mathrm{S} 8.1$ and Figure $\mathrm{S} \mathrm{8.2,} \mathrm{top} \mathrm{right)} \mathrm{resulted} \mathrm{in}$ much better correspondence between the TCSPC data and the fit. The residuals (Figure S 8.1 and Figure S 8.2 , bottom right) also show less structure and reduced amplitudes. The presence of two exponential terms in the fits makes physical sense, given the presence of a subpopulation of monomers in the aggregate solutions as identified in Section S5. In the case of the J-dimer samples, the fit returned $1.3 \mathrm{~ns}$ for the long time constant, consistent with this subpopulation of monomers. In the case of the H-tetramer samples, given the small amplitude of this component in the overall decay, we fixed the lifetime to $1.3 \mathrm{~ns}$. The agreement between the data and the biexponential fits is excellent (Figure S 8.1 and Figure S 8.2, right panels). We also compared the relative goodness of the fits by evaluating the residual sum of squares (RSS) of the monoand biexponential fits. For the J-dimer, the RSS was reduced by greater than a factor of 40 upon fitting with a biexponential. For the H-tetramer, the RSS was reduced by greater than a factor of 11 . The RSS values for the fits in Figure S 8.1 and Figure S 8.2 are found below in Table S 8.1. 
Table S 8.1. Residual Sum of Squares values for single exponential and biexponential fits to the TCSPC data for the J-dimer and $H$-tetramer

\begin{tabular}{|c|c|c|}
\hline Construct & RSS (single exponential) & RSS (bie xponential) \\
\hline J-dimer & 1.22 & 0.0298 \\
\hline H-tetramer & 0.124 & 0.0109 \\
\hline
\end{tabular}

Given the better agreement between the data and the biexponential fits, as well as the fact that a subpopulation of monomers was identified in the aggregate solutions in Section S5, we conclude that biexponential fits to the TCSPC decays are both mathematically and physically justified. 


\section{Supporting Information S.9:}

\section{Derivation of Expected Fluorescence Decay Rate for J-Aggregates}

In the limit that the nonradiative decay rate of $\mathrm{Cy} 5$ does not change upon aggregation, an expected lifetime (i.e., fluorescence decay rate) was determined for the Cy5 J-dimer. This required first determining the radiative and nonradiative decay rates for the $\mathrm{Cy} 5$ monomer, and subsequently making an assumption according to exciton theory as to the maximum extent to which the radiative decay rate could be enhanced for the J-dimer.

The radiative and nonradiative decay rates for the Cy5 monomer were derived from the experimentally determined lifetime and fluorescence quantum yields. That is,

$$
\begin{gathered}
k_{\mathrm{obs}}=k_{\mathrm{r}}+k_{\mathrm{nr}} \\
\text { and } \\
\Phi_{\mathrm{F}}=k_{\mathrm{r}} /\left(k_{\mathrm{r}}+k_{\mathrm{nr}}\right),
\end{gathered}
$$

which yields upon substitution of the first equation into the second:

$$
\Phi_{\mathrm{F}}=k_{\mathrm{r}} / k_{\mathrm{obs}}
$$

Solving the above expression for $k_{\mathrm{r}}$, we have:

$$
k_{\mathrm{r}}=\Phi_{\mathrm{F}} \times k_{\mathrm{obs}},
$$

or alternatively in terms of the experimentally observed lifetime, $\tau_{\mathrm{obs}}$ :

$$
k_{\mathrm{r}}=\Phi_{\mathrm{F}} / \tau_{\mathrm{obs}}
$$

Finally, solving the initial expression for $k_{\mathrm{nr}}$, we have:

$$
k_{\mathrm{nr}}=k_{\mathrm{obs}}-k_{\mathrm{r}}
$$

With these expressions and the experimentally determined lifetime and fluorescence quantum yield of the monomer, we can determine the radiative and nonradiative decay rates for the Cy5 monomer. Given that the monomer lifetime is ca. $1.3 \mathrm{~ns}$, the corresponding decay rate is $7.7 \times 10^{8} \mathrm{~s}^{-1}$. With this decay rate and the monomer fluorescence quantum yield of 0.29 , we determine a radiative decay rate of $2.2 \times 10^{8} \mathrm{~s}^{-1}$ (equivalent to a radiative lifetime of $4.5 \mathrm{~ns}$ ). With the observed and radiative decay rates of $7.7 \times 10^{8} \mathrm{~s}^{-1}$ and $2.2 \times 10^{8} \mathrm{~s}^{-1}$, respectively, we determine the nonradiative decay rate for the Cy5 monomer to be $5.5 \times$ $10^{8} \mathrm{~s}^{-1}$ (corresponding to a nonradiative decay time constant of $1.8 \mathrm{~ns}$ ). These parameters are summarized below in Table S 9.1. 
Table S 9.1. Observed, Radiative, and Nonradiative Decay Rates and Time Constants for the Cy5 monomer

\begin{tabular}{|l|c|c|}
\hline & Rate Constant $\boldsymbol{k}_{\boldsymbol{n}}\left(\mathbf{s}^{-1}\right)$ & Time Constant $\boldsymbol{\tau}_{\boldsymbol{n}}(\mathbf{n s})$ \\
\hline Observed & $7.7 \times 10^{8}$ & 1.3 \\
\hline Radiative & $2.2 \times 10^{8}$ & 4.5 \\
\hline Nonradiative & $5.5 \times 10^{8}$ & 1.8 \\
\hline
\end{tabular}

Given that the radiative rate of the monomer, as derived above, is ca. $2.2 \times 10^{8} \mathrm{~s}^{-1}$ (or $4.5 \mathrm{~ns}$ ), and given that for a dimer we can expect the radiative rate to increase by at most a factor of 2 (see, e.g., ref. 5), we determine a maximum radiative decay rate for the J-dimer (in the limit of full superradiance) of ca. 4.4 $\times 10^{8} \mathrm{~s}^{-1}$ (or alternatively, a minimum radiative lifetime $2.3 \mathrm{~ns}$ ). If we further assume that the nonradiative decay rate is unchanged between the monomer and $\mathrm{J}$-dimer, we arrive at an expected maximum decay rate for the J-dimer of $9.9 \times 10^{8} \mathrm{~s}^{-1}$ (or $\tau=1.0 \mathrm{~ns}$ ). 


\section{Supporting Information S.10:}

\section{Transient Absorption Pulse Characterization}

\subsection{Pulse duration}

An autocorrelation was performed via polarization gating methods ${ }^{10}$ to measure the pulse duration at the sample position in the transient absorption spectrometer. In brief, the measurement was performed by first setting the relative pump and probe polarizations to 45 degrees at the sample position. A cube analyzer polarizer was then placed immediately after the sample position to null the probe signal on the $\mathrm{Si}$ photodiode detector in the absence of the pump beam. The energy density and wavelengths of the pump and probe beams were both set to $1 \mathrm{~mJ} / \mathrm{cm}^{2}$ and $640 \mathrm{~nm}$, respectively, and the pump and probe beams were focused into a $2 \mathrm{~mm}$ path length quartz cuvette containing distilled water. The relative pump and probe time delay was controlled with a mechanical delay stage (see Section S2.3.2 and Figure S 2.1). Over the course of the autocorrelation, the pulses experienced both spatial and temporal overlap at the sample position. In the presence of the pump (i.e., during temporal overlap), the probe experienced polarization rotation due to the optical Kerr effect, resulting in some of the probe pulse transmitting through the analyzer polarizer to the photodiode. The resulting autocorrelation pulse shape is shown in Figure S 10.1 (black trace). The full width at half $\max (\mathrm{FWHM})$ for the Gaussian fit (red trace) is $760 \mathrm{fs}$.

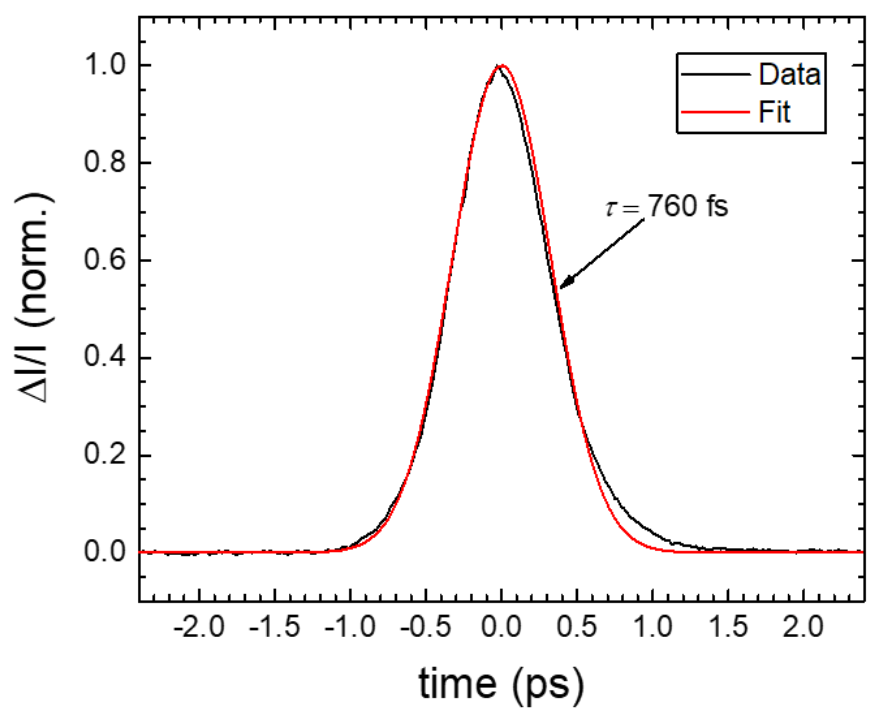

Figure S 10.1. The measured temporal intensity profile (black) and Gaussian fit (red) of the autocorrelation of the pump and probe pulses present at the sample position in the transient absorption spectrometer. 


\section{Supporting Information S.11}

\section{Comparison of Sample Absorption Spectra and Laser Spectra}

The laser was tuned to excite and probe the most intense absorption feature of each sample. Figure S 11.1 shows the absorption spectra of representative sample solutions plotted as solid lines; associated representative laser pulse spectra are plotted as dashed lines of the same color.

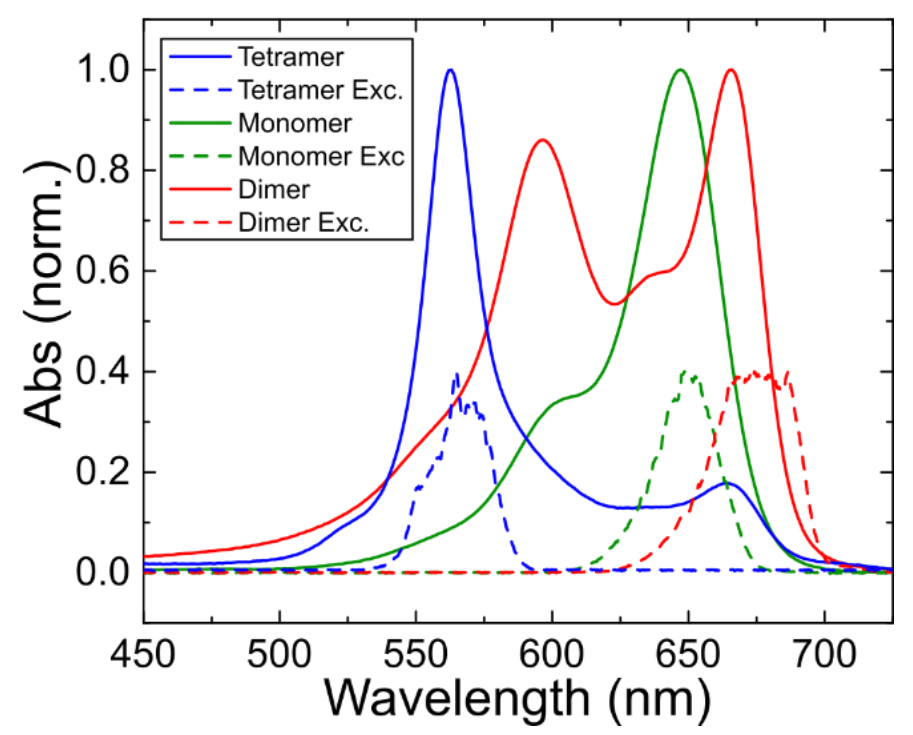

Figure S 11.1. Absorption (solid) and representative laser pulse spectra (dashed) for the H-tetramer (blue), Cy5 monomer (green), and J-dimer (red) samples. 


\section{Supporting Information S.12:}

\section{Ground State Recovery of Aggregates is Independent of Excitation Wavelength}

\subsection{H-Tetramer}

The H-tetramer transient absorption decays discussed in the main text were collected with the light source tuned to excite the higher energy (allowed) electronic transition at $563 \mathrm{~nm}$. While it is normally preferable in time-resolved measurements to excite the lowest energy electronic transition, for the $\mathrm{H}$ tetramer solution, this approach presented complicating factors that would reduce the accuracy of measuring the ground-state recovery time constant. These factors are: (i) the suppressed absorption of the low energy electronic transition due to $\mathrm{H}$-aggregation, and (ii) the large spectral overlap of the $\mathrm{H}$-tetramer absorption with the J-dimer $\left(\lambda_{\max }=666 \mathrm{~nm}\right)$ and monomer $\left(\lambda_{\max }=647 \mathrm{~nm}\right)$ absorption at long wavelengths. Irrespective of these complicating factors, the excited state lifetime of the H-tetramer as measured by TA should be independent of the excitation wavelength since excitation of the high energy electronic state is expected to result in ultrafast internal conversion to the lowest-energy electronic state (per Kasha's rule ${ }^{11}$ ), followed by comparatively slower relaxation to the ground state according to the intrinsic photophysics of the aggregate.

Nonetheless, TA measurements exciting the H-tetramer at its lowest energy transition were performed to confirm the above assumption. The TA light source was tuned to $700 \mathrm{~nm}$ in order to maximize contrast between the H-tetramer population and the overlapping monomer and J-dimer subpopulations. In order to obtain an optical density sufficient for TA measurements, a highly concentrated $(10 \mu \mathrm{M}) \mathrm{H}$ tetramer solution was prepared. Given that high concentrations favor the H-tetramer subpopulation, ${ }^{1}$ and the monomer absorption at the $700 \mathrm{~nm}$ excitation and probing wavelength is minimal (Supporting Information S.4: Monomer Photophysics), the solution used for these wavelength dependence studies of the H-tetramer was not further subjected to PAGE purification.

Figure S 12.1 shows the averaged results of TA measurements on the H-tetramer upon excitation at $565 \mathrm{~nm}$ (left) and $700 \mathrm{~nm}$ (right); the corresponding fitting parameters are displayed in Table S 12.1. The TA decay for the solution excited at $700 \mathrm{~nm}$ was fit with a triexponential function that yielded three time constants corresponding to the H-tetramer, J-dimer, and monomer contributions to the TA signal. We assign the shortest time constant ( $8 \mathrm{ps}$ ) to the J-dimer subpopulation and the $39 \mathrm{ps}$ time constant to the H-tetramer. The TA decay for the solution excited at $565 \mathrm{~nm}$ was fit with a biexponential function and yielded a shorttime constant of $35 \mathrm{ps}$ that corresponded to the H-tetramer. For both the bi- and tri-exponential fits, the longest time constant, which corresponds to the monomer sub-population, was fixed at 1.5 ns. Critically, the time constants of $35 \mathrm{ps}$ and $39 \mathrm{ps}$ for the H-tetramer component of the TA decays determined at 565 and $700 \mathrm{~nm}$, respectively, are in good agreement, indicating that relaxation from high-lying excited states due to excitation at short wavelength does not complicate interpretation of the intrinsic ground-state recovery time of the $\mathrm{H}$-tetramer. 


\section{H Tetramer}

$565 \mathrm{~nm}$ exc.

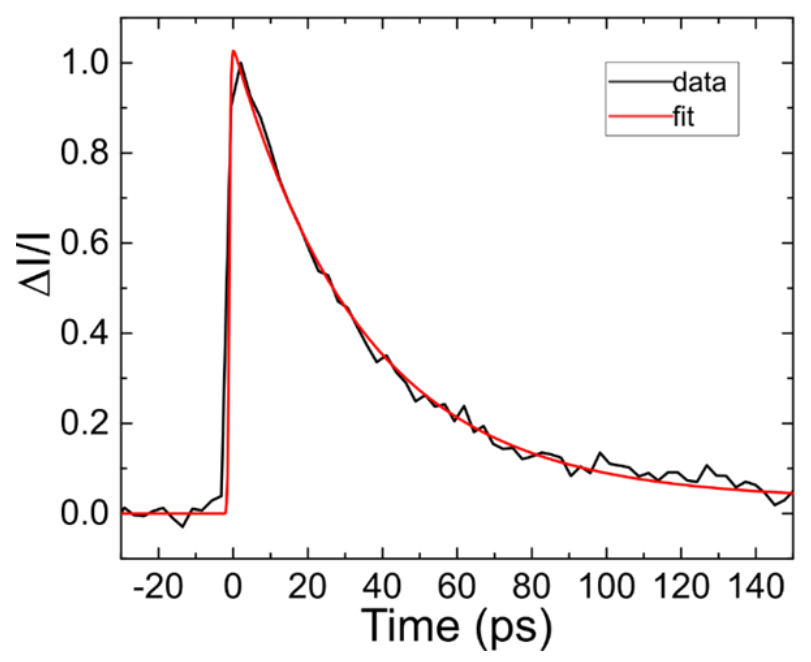

$700 \mathrm{~nm}$ exc.

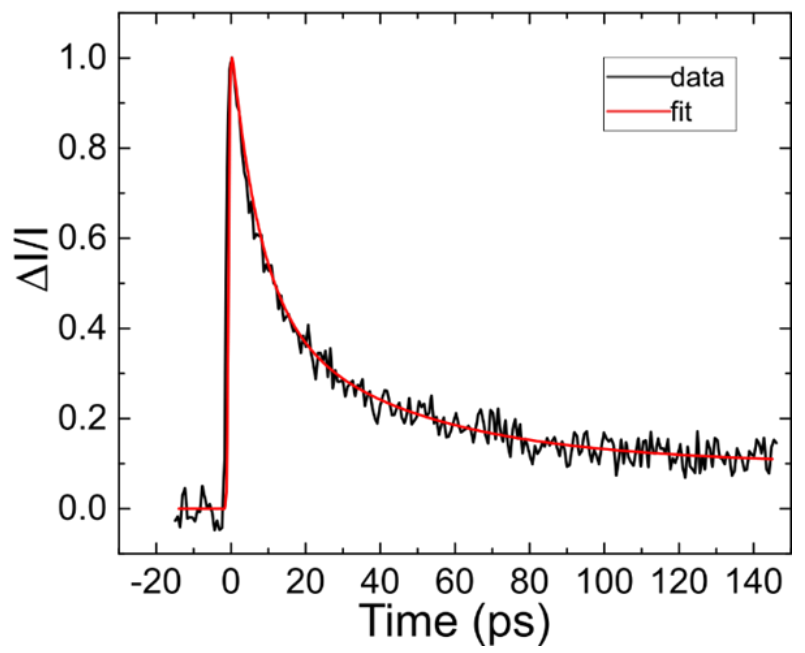

Figure S 12.1. Left: Transient absorption decay of a PAGE purified H-tetramer solution with the light source tuned to $565 \mathrm{~nm}$. Right: Transient absorption decay of a highly concentrated H-tetramer solution with the light source tuned to $700 \mathrm{~nm}$.

Table S 12.1. TA Fitting Parameters for H-tetramer Wavelength Dependence Studies. $A_{n}$ represents the pre-exponential weighting factor and $\tau_{n}$ represents the fitted time constant.

\begin{tabular}{|c|c|c|c|c|c|c|}
\hline$\lambda_{\text {exc }}(\mathbf{n m})$ & $\mathbf{A}_{\mathbf{1}}$ & $\boldsymbol{\tau}_{\mathbf{1}}(\mathbf{p s})$ & $\mathbf{A}_{\mathbf{2}}$ & $\boldsymbol{\tau}_{\mathbf{2}}(\mathbf{p s})$ & $\mathbf{A}_{\mathbf{3}}$ & $\boldsymbol{\tau}_{\mathbf{3}}$ (ns) \\
\hline 565 & N/A & N/A & 0.97 & 35 & 0.03 & 1.5 (fixed) \\
\hline 700 & 0.56 & 8 & 0.34 & 39 & 0.10 & 1.5 (fixed) \\
\hline
\end{tabular}

\subsection{J-dimer}

The wavelength dependence of the transient absorption kinetics of the J-dimer solution was tested to evaluate what impact structural heterogeneity may have, if any, on the ground state recovery kinetics of the J-dimer (Figure 2).

The basis for performing such a measurement is that a minor population of H-aggregates was determined to be present in the J-dimer solution (Supporting Information S.3) and the presence of structural heterogeneity could conceivably alter the kinetics measured at $675 \mathrm{~nm}$ such that they are not representative of the intrinsic lifetime of the 'pure' J-dimer. In Supporting Information S.3, the minor population of Haggregates was found to absorb the strongest at ca. $595 \mathrm{~nm}$, which overlaps the high energy excitonic $\left(S_{1} S_{0}\right)$ ' "band of the J-dimer. Accordingly, the transient absorption decay of the PAGE-purified J-dimer solution was measured with the light source set to $600 \mathrm{~nm}$ to determine its excited-state lifetime.

Figure S 12.2 displays a biexponential fit to the transient absorption decay measured at $600 \mathrm{~nm}$ (left-hand side) and the associated residual (right-hand side). For the biexponential fitting function, one time constant was fixed to that of the monomer $-1.5 \mathrm{~ns}$ (based on the results of several preceding supporting sections - Supporting Information S.4, Supporting Information S.5, Supporting Information S.8) - and the 
other time constant was allowed to float, returning a value of $12 \mathrm{ps}$ and the majority of the amplitude (94.5\%). Figure S 12.3 displays a triexponential fit to the same data (left-hand side) and associated residual (right-hand side). Two of the three time constants of the triexponential fitting function were fixed: (i) a long time constant, fixed at $1.5 \mathrm{~ns}$ to account for the monomer subcomponent, and (ii) a short time constant, fixed at the $11 \mathrm{ps}$ measured for the $\mathrm{J}$-dimer at $675 \mathrm{~nm}$. For the time constant that was allowed to float, the triexponential fit returned a value of $74 \mathrm{ps,} \mathrm{contributing} 12.7 \%$ of the total signal. A summary of the fit parameters and fit goodness benchmarks is provided in Table $\mathrm{S}$ 12.2.

PAGE Purified J-Dimer $600 \mathrm{~nm}$
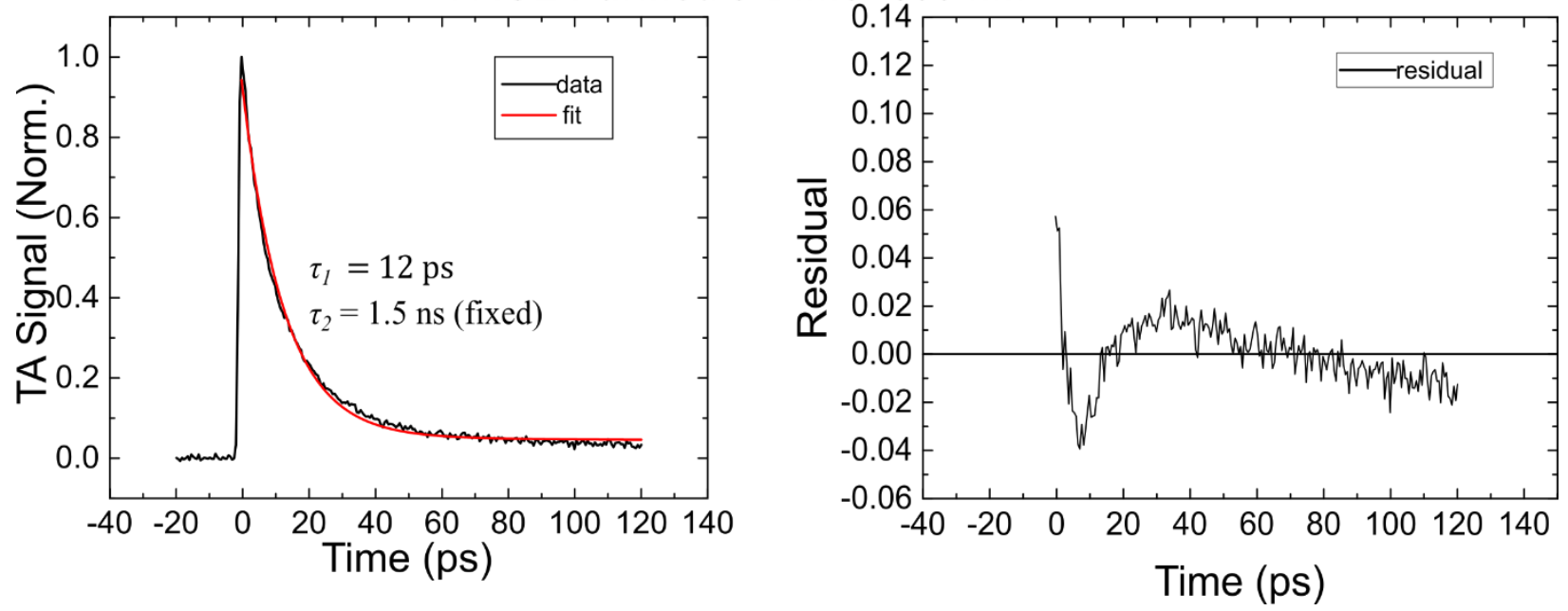

Figure S 12.2. Left: Transient absorption decay (black) and biexponetial fit (red) of a PAGE purified J-dimer solution measured at $600 \mathrm{~nm}$ (black). Right: Associated residual between the fit and the data.

PAGE Purified J-Dimer 600 nm
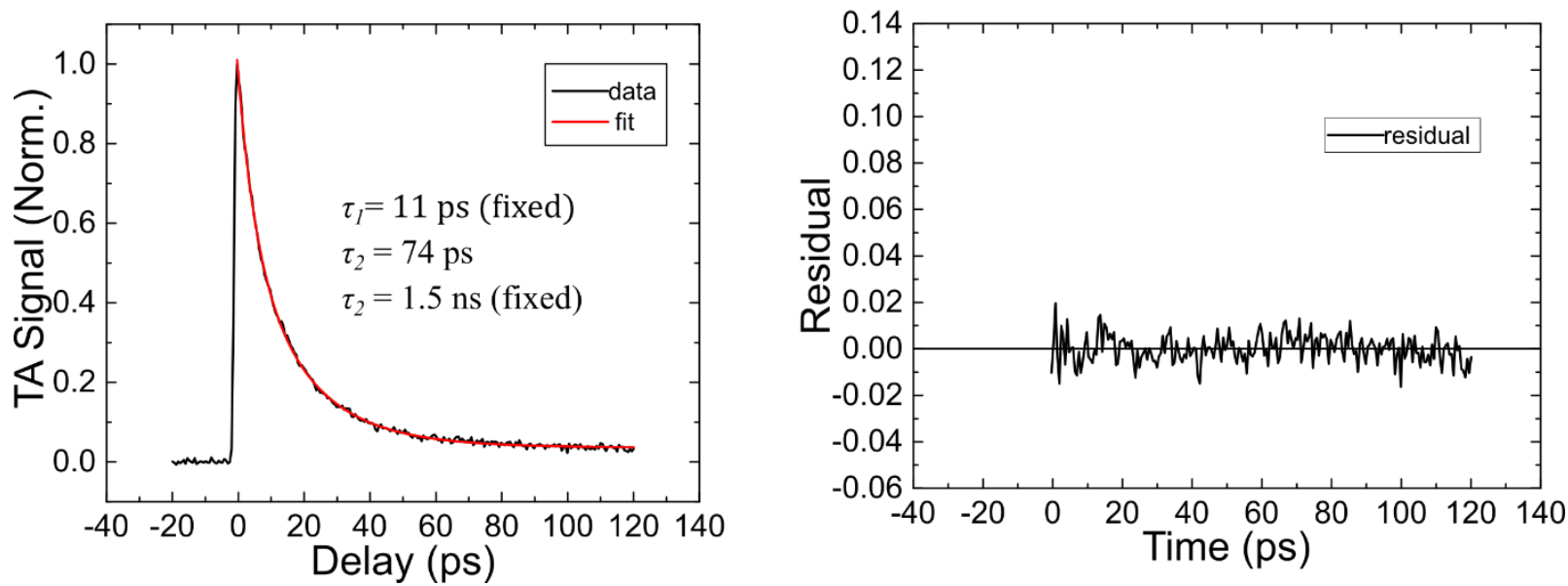

Figure S 12.3. Left: Transient absorption decay (black) and triexponential fit (red) of a PAGE purified J-dimer solution measured at $600 \mathrm{~nm}$ (black). Right: Associated residual between the fit and the data. 
Table S 12.2. Fitting parameters, R-squared, and Residual Sum Squared (RSS) values for the TA decay of the J-dimer solution as measured at $600 \mathrm{~nm}$. Biexponential and triexpontial fits are included for comparing goodness of fits.

\begin{tabular}{|c|c|c|c|c|c|c|c|c|}
\hline $\begin{array}{c}\lambda_{\text {exc }} \\
(\mathbf{n m})\end{array}$ & $\mathbf{A}_{\mathbf{1}}$ & $\boldsymbol{\tau}_{\mathbf{1}}(\mathbf{p s})$ & $\mathbf{A}_{\mathbf{2}}(\%)$ & $\boldsymbol{\tau}_{\mathbf{2}}(\mathbf{p s})$ & $\mathbf{A}_{\mathbf{3}}(\boldsymbol{\%})$ & $\boldsymbol{\tau}_{\mathbf{3}}(\mathbf{n s})$ & $\begin{array}{c}\text { R- } \\
\text { Squared }\end{array}$ & $\begin{array}{c}\text { Residual sum } \\
\text { squared }(\mathrm{RSS})\end{array}$ \\
\hline 600 & N/A & N/A & 94.5 & 12 & 5.5 & 1.5 (fixed) & 0.994 & 0.042 \\
\hline 600 & 86.3 & 11 (fixed) & 12.7 & 74 & 0.9 & 1.5 (fixed) & 0.997 & 0.008 \\
\hline
\end{tabular}

A visual comparison of the fits and residuals from Figures S 12.2 and S12.3 indicates that the triexponential fit better represents the TA decay measured at $600 \mathrm{~nm}$. Specifically, the triexponential fit residual shows no structure, while the biexponential fit residual alternately overestimates and underestimates the TA signal. Further, the R-squared and residual sum squared (RSS) values for the triexponential fit are improved over the biexponential fit.

The results indicate that an additional decay component is present in the TA kinetics of the $\mathrm{J}$-dimer solution measured at $600 \mathrm{~nm}$. We assign this additional decay component to the ground state recovery of the minor H-aggregate population identified in Supporting Information S.3. The ground state recovery kinetics of the $\mathrm{H}$-aggregate component absorbing at ca. $595 \mathrm{~nm}$ are nearly an order-of-magnitude $(\sim 7 \times)$ slower than that of the J-dimer. The slower ground-state recovery kinetics compared with the J-dimer is the same trend observed for the $\mathrm{H}$-tetramer that absorbs most strongly at ca. $563 \mathrm{~nm}$, which had a lifetime of ca. 35 ps. Thus, the analysis has returned an additional $\mathrm{H}$-aggregate component whose ground-state recovery is governed by nonradiative decay.

For comparison with the TA kinetics for the J-dimer solution measured at $600 \mathrm{~nm}$ (Table S 12.2), Table S 12.3 displays the TA kinetics for the J-dimer solution measured at $675 \mathrm{~nm}$.

Table S 12.3. Biexponential fitting parameters, R-squared, and Residual Sum Squared (RSS) values for the TA decay of the Jdimer solution as measured at $675 \mathrm{~nm}$.

\begin{tabular}{|c|c|c|c|c|c|c|}
\hline $\begin{array}{c}\lambda_{\text {exc }} \\
(\mathbf{n m})\end{array}$ & $\mathbf{A}_{\mathbf{1}}(\%)$ & $\tau_{1}(\mathbf{p s})$ & $\mathbf{A}_{2}(\%)$ & $\tau_{2}(\mathbf{n s})$ & $\begin{array}{c}\text { R- } \\
\text { Squared }\end{array}$ & $\begin{array}{c}\text { Residual sum } \\
\text { squared }(\boldsymbol{R S S})\end{array}$ \\
\hline 675 & 97.1 & 11 & 2.9 & 1.5 (fixed) & 0.998 & 0.012 \\
\hline
\end{tabular}

Clearly, the data at $675 \mathrm{~nm}$ are well modeled by the bi-exponential fit (Figure 2 and Table S 12.3). Given that the TA kinetics for the J-dimer solution measured at $675 \mathrm{~nm}$ do not warrant inclusion of a third component, we conclude that the short time constant measured for the sample under these conditions is that of the 'pure' J-dimer. This is not unexpected, because $675 \mathrm{~nm}$ is far from the ca. $595 \mathrm{~nm}$ absorption maximum of the H-aggregate determined in Supporting Information S.3. Thus, we conclude that the presence of such structural heterogeneity does not complicate our interpretation that the short time constant measured in the TA kinetics at $675 \mathrm{~nm}$ represents the intrinsic lifetime of the 'pure' J-dimer. 


\section{Supporting Information S.13:}

\section{Ground State Recovery of Aggregates is Independent of Incident Pump Fluence}

The power (pump fluence) dependence of the ground state recovery was investigated with transient absorption spectroscopy. Solutions of the J-dimer and H-tetramer were excited at 675 and $565 \mathrm{~nm}$, respectively, and their transient absorption decays were monitored. The value of $\tau_{1}$ was compared for multiple beam intensities for each sample.

\subsection{J-dimer}

The dependence of the ground state recovery time constant of the J-dimer on excitation intensity as measured by transient absorption spectroscopy was investigated by measuring the ground state recovery time constant at differing excitation energy intensities. The J-dimer solution was excited at $675 \mathrm{~nm}$ and the transient absorption decay was measured for pump intensities of: $487 \mu \mathrm{J} / \mathrm{cm}^{2}, 1.2 \mathrm{~mJ} / \mathrm{cm}^{2}, 2.1 \mathrm{~mJ} / \mathrm{cm}^{2}$, and $4.2 \mathrm{~mJ} / \mathrm{cm}^{2}$. The probe fluence was held below $250 \mu \mathrm{J} / \mathrm{cm}^{2}$ for all measurements. Figure $\mathrm{S} 13.1$ displays an overlay of the J-dimer transient absorption decays for each of the incident pump fluences. The transient absorption decay traces lie on top of one another, indicating no additional decay pathways become active in the J-dimer at higher pump intensities.

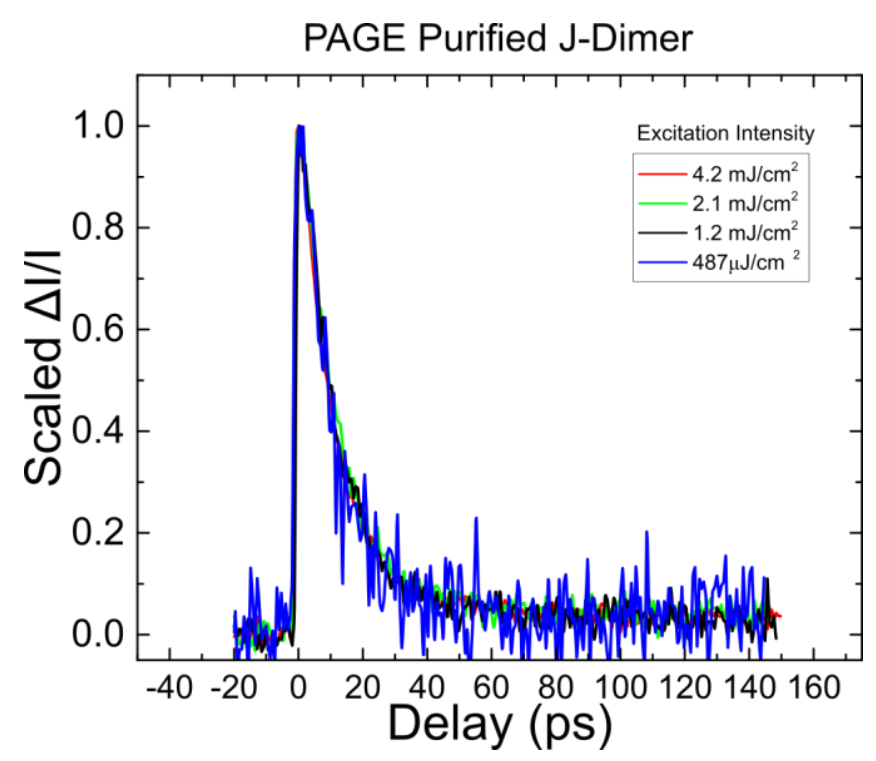

Figure S 13.1. Fluence dependence of J-dimer transient absorption. Excitation and probing wavelength was $675 \mathrm{~nm}$.

\section{$13.2 \quad$ H-Tetramer}

For the H-tetramer solution, the light source was tuned to $565 \mathrm{~nm}$ and the transient absorption decay was measured at pump fluences of $340 \mu \mathrm{J} / \mathrm{cm}^{2}, 800 \mu \mathrm{J} / \mathrm{cm}^{2}$, and $1.3 \mathrm{~mJ} / \mathrm{cm}^{2}$. The probe fluence was held below at $250 \mu \mathrm{J} / \mathrm{cm}^{2}$ for all measurements. Figure S 13.2 shows an overlay of the transient absorption decay for each pump intensity. As was observed with the J-dimer, the TA decay traces overlap for all pump intensities, differing only in noise amplitude. We therefore conclude that no additional decay pathways are activated in the H-tetramer at high pump fluences. 


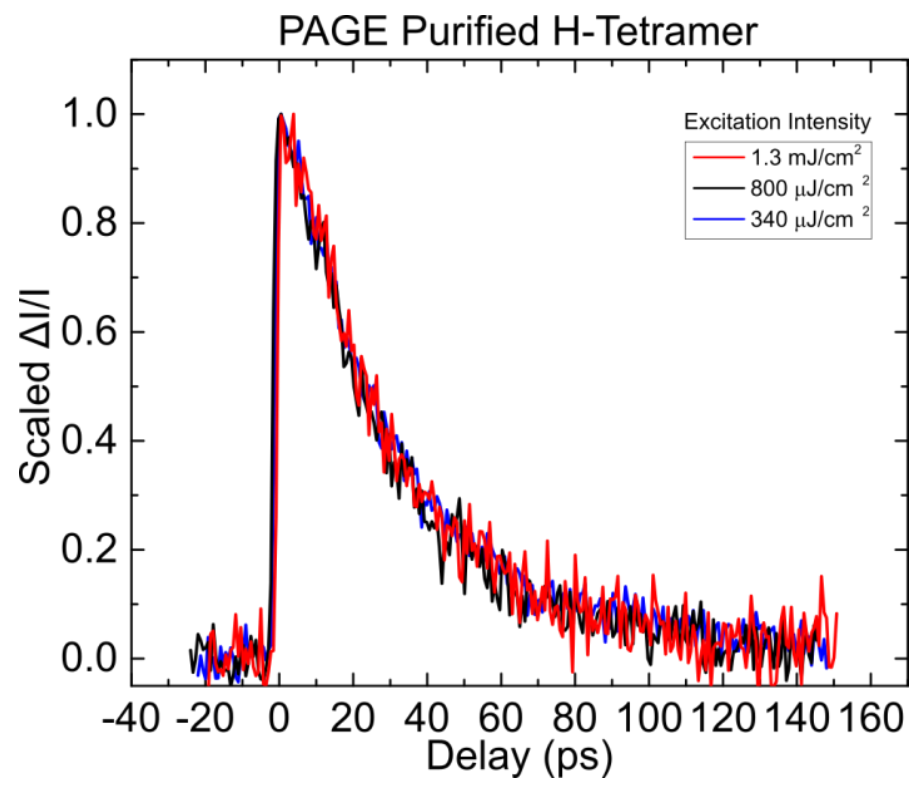

Figure S 13.2. Fluence dependence of H-tetramer transient absorption. Excitation and probing wavelength was $565 \mathrm{~nm}$. 


\section{Supporting Information S.14:}

\section{Quantitative Analysis of Radiative and Nonradiative Rates}

In this section, we perform a quantitative analysis of the kinetic results to determine the extent to which nonradiative decay contributes to the overall decay of the monomer and aggregates. We adopt a similar procedure as that reported in Supporting Information S.9. Namely, the radiative and nonradiative decay rates for the different samples were derived from the experimentally determined lifetime as measured by transient absorption spectroscopy (see Figure 4 of the main text) while also utilizing the fluorescence quantum yield of the monomer (see Supporting Information S.4). That is,

$$
\begin{gathered}
k_{\mathrm{obs}}=k_{\mathrm{r}}+k_{\mathrm{nr}} \\
\text { and } \\
\Phi_{\mathrm{F}}=k_{\mathrm{r}} /\left(k_{\mathrm{r}}+k_{\mathrm{nr}}\right),
\end{gathered}
$$

which yields upon substitution of the first equation into the second:

$$
\Phi_{\mathrm{F}}=k_{\mathrm{r}} / k_{\mathrm{obs}}
$$

Solving the above expression for $k_{\mathrm{r}}$, we have:

$$
k_{\mathrm{r}}=\Phi_{\mathrm{F}} \times k_{\mathrm{obs}},
$$

or alternatively in terms of the experimentally observed lifetime, $\tau_{\mathrm{obs}}$ :

$$
k_{\mathrm{r}}=\Phi_{\mathrm{F}} / \tau_{\mathrm{obs}}
$$

Finally, with knowledge of $k_{\mathrm{r}}$ and solving the initial expression for $k_{\mathrm{n}}$, we have:

$$
k_{\mathrm{nr}}=k_{\mathrm{obs}}-k_{\mathrm{r}} .
$$

The extent to which nonradiative decay contributes to the overall decay is evaluated as:

$$
k_{\mathrm{nr}} / k_{\mathrm{obs}} \cdot
$$

The results of the above analysis are presented in Table S 14.1 below. 
Table S 14.1. Fluorescence Quantum Yields, Overall Lifetimes, and Overall, Radiative, and Nonradiative Decay Rates for Cy5 MonomerA, J-dimers, and H-tetramers. Note that a single monomer species was analyzed here.

\begin{tabular}{|l|l|l|l|l|l|l|}
\hline Sample & $\boldsymbol{\Phi}_{\mathbf{F}}$ & $\tau_{\text {obs }}(\mathbf{n s})$ & $\boldsymbol{k}_{\mathbf{o b s}}\left(\mathbf{s}^{-1}\right)$ & $\boldsymbol{k}_{\mathbf{r}}\left(\mathbf{s}^{-1}\right)$ & $\boldsymbol{k}_{\mathbf{n r}}\left(\mathbf{s}^{-1}\right)$ & $\boldsymbol{k}_{\mathbf{n r}} / \boldsymbol{k}_{\mathbf{o b s}}(\mathbf{\%})$ \\
\hline MonomerA & 0.29 & 1.3 & $7.69 \times 10^{8}$ & $2.23 \times 10^{8}$ & $5.46 \times 10^{8}$ & 71.00 \\
\hline J-dimer $(\mathrm{N}=1.5)$ & N/A & 0.011 & $9.09 \times 10^{10}$ & $3.34 \times 10^{8}$ & $9.06 \times 10^{10}$ & 99.63 \\
\hline H-tetramer & N/A & 0.035 & $2.86 \times 10^{10}$ & $1.12 \times 10^{7}$ & $2.86 \times 10^{10}$ & 99.96 \\
\hline
\end{tabular}

Note that the radiative decay rate constants for the $\mathrm{J}$-dimer and $\mathrm{H}$-tetramer were approximated as $k_{r, J}=1.5 \times k_{r, m}$ and $k_{r, H}=0.05 \times k_{r, m}$. The basis for the estimate of $k_{r, J}$ is explained in more detail in Supporting Information S.6, specifically in section S6.2.2. The estimate for $k_{r, H}$ is based on the consideration that the extinction of the lowest-energy band of the H-aggregate is approximately $20 \times$ less than that of the monomer. ${ }^{8}$ Clearly, as shown by the $k_{\mathrm{nr}} / k_{\text {obs }}$ percentages in the last column of Table $\mathrm{S} 14.1$, nonradiative decay is the primary relaxation pathway in the aggregates. 


\section{Supporting Information S.15:}

\section{Charge Transfer is Ruled Out as a Nonradiative Decay Mechanism}

In this supporting section, we consider the likelihood that charge transfer is primarily responsible for nonradiative decay in the DNA-templated Cy5 aggregates.

First, we consider the fundamental likelihood of charge transfer between the Cy5 molecules in the DNA-templated aggregates. In a low dielectric environment, such as an assembly of Cy5 molecules, charge transfer between the Cy5 molecules is considered highly unlikely because:(i) the molecules are the same and so there is no driving force for electron transfer, and (ii) the barrier to electron transfer is exceptionally large, resulting in binding energies $>>k T .{ }^{12,13}$ Thus, charge transfer between Cy5 molecules in an assembly of Cy5 molecules is considered unlikely. We cannot ignore, however, that the environment also comprises DNA and water. Considering that the environment also includes DNA and water is important because a special case of charge transfer between an assembly of two (or more) of the same conjugated organic molecules can occur when the molecules are dissolved in polar solvents. ${ }^{14}$ Such a process has been colloquially named symmetry-breaking charge transfer (SB CT) and has been observed to occur, to varying extents, in 9,9'-bianthryl, ${ }^{15}$ a perylene derivative, ${ }^{16}$ various perylene diimide derivatives, ${ }^{17-20}$ a naphthalene diimide derivative, ${ }^{21}$ and a diketopolypyrrole derivative. ${ }^{22}$ In this case, solvation dynamics drive the CT process, ${ }^{14}$ which otherwise would not occur. Thus, rates of SB CT generally range from $100 \mathrm{~s}$ of fs to $100 \mathrm{~s}$ of ps, followed by slower rates of back CT (per the expectations of Marcus theory). ${ }^{14}$ Given that our DNAtemplated Cy5 aggregates are dissolved in aqueous buffer, SB CT is a possible nonradiative decay mechanism.

We proceed to discuss how the transient absorption (TA) kinetics observed for the DNA-templated Cy5 aggregate solutions (Figure 4) can inform whether SB CT is a plausible nonradiative decaymechanism. As noted above, for SB CT, a two-step process is anticipated. ${ }^{14}$ In the case of weak coupling between molecules, we expect: (i) the dissociation of the initial excitation, i.e., forward CT, and (ii) recovery of the system to the ground state via back CT. In a TA experiment, such a two-step SB CT process is expected to be manifested in the form of bi-exponential kinetics. The first kinetic component is expected because the electronic structure of the system changes as the initially neutral exciton dissociates into anion and cation, and the second kinetic component is expected as the system recovers back to the ground state via back CT. Figure 4 shows that, while a small amplitude (less than 5\%) component is apparent at long time delays arising from the parallel decay of a subpopulation of monomers (Supporting Information S.5 and S.8), a single exponential function suffices to model the ground-state recovery kinetics of the DNA-templated Cy5 aggregates at early time delays (i.e., sub-100-ps). Since we do not observe bi-exponential kinetics associated with the DNA-templated Cy5 aggregates, we therefore rule out SB CT of this form.

One could argue that SB CT of this form is not expected for the DNA-templated Cy5 aggregates of the present study, for which strong electronic coupling between molecules is apparent (Figure 2). That is, in the limit of strong electronic coupling between molecules, where the assembly of molecules behaves as a single chromophore, SB CT is expected to result in an excited state with partial CT character, i.e., a so-called CT state, rather than full CT. ${ }^{14,22}$ In the case of SB CT in the limit of strong electronic coupling between molecules, two-step kinetics are still expected. In this case, the initial excitation first relaxes to a state with appreciable CT character, which subsequently relaxes to the ground state. The model in the 
limiting case of strong electronic coupling between molecules further predicts that the resultant CT state should decay comparatively slowly with respect to the corresponding more neutral initial excitation, whose lifetime can be approximated as that of the monomer. Because CT states have some admixture of chargeresonance configurations in their overall wavefunction, and because the optical allowed-ness of the transition decreases with the newly introduced CT character, the excited-state lifetime of a CT state generally increases with respect to that of a more neutral excitation. This is what is observed for 9,9'bianthryl, a classical SB CT compound. ${ }^{15}$ Given that we observe the excited-state lifetime to drastically decrease in our DNA-templated Cy5 aggregates (Figure 4), as opposed to increase as would be expected for a CT state, we further rule out SB CT in the limit of strong electron coupling as a possible nonradiative decay mechanism.

Based on the discussion above, namely that the observed TA kinetics do not support a model of charge transfer between Cy5 molecules in either weak or strong coupling limits of SB CT, we rule out charge transfer as a nonradiative decay mechanism for the DNA-templated Cy5 aggregates studied in this work. 


\section{$\underline{\text { References }}$}

(1) Cannon, B. L.; Kellis, D. L.; Patten,L. K.; Davis, P. H.; Lee, J.; Graugnard, E.; Yurke, B.; Knowlton, W. B. Coherent Exciton Delocalization in a Two-State DNA-Templated Dye Aggregate System. $J$. Phys. Chem. A 2017, 121 (37), 6905-6916.

(2) Rurack, K.; Spieles, M. Fluorescence Quantum Yields of a Series of Red and Near-Infrared Dyes Emitting at 600-1000 Nm. Anal. Chem 2011, 83, 1232-1242.

(3) Lakowicz, J. R. Principles of Fluorescence Spectroscopy, 3rd ed.; Springer: New York, NY, 2006.

(4) Hestand, N. J.; Spano, F. C. Molecular Aggregate Photophysics beyond the Kasha Model: Novel Design Principles for Organic Materials. Acc. Chem. Res. 2017, 50 (2), 341-350.

(5) Muenter, A. A.; Brumbaugh, D. V; Apolito, J.; Horn, L. A.; Spano, F. C.; Mukamel, S. Size Dependence of Excited-State Dynamics for J-Aggregates at AgBr Interfaces. J. Phys. Chem. 1992, $96(7), 2783-2790$.

(6) Spano, F. C.; Kuklinski, J. R.; Mukamel, S. Cooperative Radiative Dynamics in Molecular Aggregates. J. Chem. Phys. 1991, 94 (11), 7534-7544.

(7) Pope, M.; Swenberg, C. E. Electronic Processes in Organic Crystals andPolymers, Second.; Oxford University Press: New York, NY, 1999.

(8) Rösch, U.; Yao, S.; Wortmann, R.; Würthner, F. Fluorescent H-Aggregates of Merocyanine Dyes. Angew. Chemie - Int. Ed. 2006, 45 (42), 7026-7030.

(9) Ryu, N.; Okazaki, Y.; Pouget, E.; Takafuji, M.; Nagaoka, S.; Ihara, H.; Oda, R. Fluorescence Emission Originated from H-Aggregated Cyanine Dye with Chiral Gemini Surfactant Assemblies Having Narrow Absorption Band and Remarkably Large Stokes Shift. Chem. Commun. 2017, 53 (63), 8870-8873.

(10) Trebino, R.; DeLong, K. W.; Fittinghoff, D. N.; Sweetser, J. N.; Krumbügel, M. A.; Richman, B. A.; Kane, D. J. Measuring Ultrashort Laser Pulses in the Time-Frequency Domain Using FrequencyResolved Optical Gating. Rev. Sci. Instrum. 1997, 68 (9), 3277-3295.

(11) Turro, N. J.; Scaiano, J. C.; Ramamurthy, V. Modern Molecular Photochemistry of Organic Molecules; University Science Books: Sausalito, Calif, 2010.

(12) Gregg, B. A. Excitonic Solar Cells. J. Phys. Chem. B 2003, 107 (20), 4688-4698.

(13) Gregg, B. A. Toward a Unified Treatment of Electronic Processes in Organic Semiconductors. $J$. Phys. Chem. B 2004, 108 (45), 17285-17289.

(14) Vauthey, E. Photoinduced Symmetry-Breaking Charge Separation. ChemPhysChem 2012,13 (8), 2001-2011.

(15) Grabowski, Z. R.; Rotkiewicz, K.; Rettig, W. Structural Changes Accompanying Intramolecular Electron Transfer: Focus on Twisted Intramolecular Charge-Transfer States and Structures. Chem. Rev. 2003, 103 (10), 3899-4032.

(16) Cook, R. E.; Phelan, B. T.; Kamire, R. J.; Majewski, M. B.; Young, R. M.; Wasielewski, M. R. 
Excimer Formation and Symmetry-Breaking Charge Transfer in Cofacial Perylene Dimers. J. Phys. Chem. A 2017, 121 (8), 1607-1615.

(17) Giaimo, J. M.; Gusev, A. V.; Wasielewski, M. R. Excited-State Symmetry Breaking in Cofacial and Linear Dimers of a Green Perylenediimide Chlorophyll Analogue Leading to Ultrafast Charge Separation. J. Am. Chem. Soc. 2002, 124 (29), 8530-8531.

(18) Holman, M. W.; Yan, P.; Adams, D. M.; Westenhoff, S.; Silva, C. Ultrafast Spectroscopy of the Solvent Dependence of Electron Transfer in a Perylenebisimide Dimer. J. Phys. Chem. A 2005, 109 (38), 8548-8552.

(19) Wu, Y.; Young, R. M.; Frasconi, M.; Schneebeli, S. T.; Spenst, P.; Gardner, D. M.; Brown, K. E.; Würthner, F.; Stoddart, J. F.; Wasielewski, M. R. Ultrafast Photoinduced Symmetry-Breaking Charge Separation and Electron Sharing in Perylenediimide Molecular Triangles. J. Am. Chem. Soc. 2015, 137 (41), 13236-13239.

(20) Sung, J.; Nowak-Król, A.; Schlosser, F.; Fimmel, B.; Kim, W.; Kim, D.; Würthner, F. Direct Observation of Excimer-Mediated Intramolecular Electron Transfer in a Cofacially-Stacked Perylene Bisimide Pair. J. Am. Chem. Soc. 2016, 138(29), 9029-9032.

(21) Banerji, N.; Fürstenberg, A.; Bhosale, S.; Sisson, A. L.; Sakai, N.; Matile, S.; Vauthey, E. Ultrafast Photoinduced Charge Separation in Naphthalene Diimide Based Multichromophoric Systems in Liquid Solutions and in a Lipid Membrane. J. Phys. Chem. B 2008, 112 (30), 8912-8922.

(22) Mauck, C. M.; Bae, Y. J.; Chen, M.; Powers-Riggs, N.; Wu, Y.-L.; Wasielewski, M. R. ChargeTransfer Character in a Covalent Diketopyrrolopyrrole Dimer: Implications for Singlet Fission. ChemPhotoChem 2018, 2 (3), 223-233. 